\title{
EXTREME VALUE THEORY AND RETURN TIME STATISTICS FOR DISPERSING BILLIARD MAPS AND FLOWS, LOZI MAPS AND LORENZ-LIKE MAPS.
}

\author{
CHINMAYA GUPTA, MARK HOLLAND, AND MATTHEW NICOL
}

\begin{abstract}
In this paper we establish extreme value statistics for observations on a class of hyperbolic systems: planar dispersing billiard maps and flows, Lozi maps and Lorenz-like maps. In particular we show that for time series arising from Hölder observations on these systems the successive maxima of the time series are distributed according to the corresponding extreme value distributions for independent identically distributed processes. These results imply an exponential law for the hitting and return time statistics of these dynamical systems.
\end{abstract}

\section{INTRODUCTION}

Suppose $\left\{X_{n}\right\}$ is a stationary stochastic process and define $\left\{M_{n}\right\}$, the sequence of successive maxima by $M_{n}:=\max \left\{X_{1}, \ldots, X_{n}\right\}$. Extreme value theory is concerned with the limiting distribution of the sequence of normalized maxima $a_{n}\left(M_{n}-b_{n}\right)$. There is a well developed theory $[22,16,30]$ assuming $\left\{X_{n}\right\}$ are independent for the limiting distribution of $\left\{M_{n}\right\}$ under linear scaling $a_{n}\left(M_{n}-b_{n}\right)$ defined by constants $a_{n}>0, b_{n} \in \mathbb{R}$. It is known that there are only three non-degenerate distributions $G(x)$ such that $\lim _{n \rightarrow \infty} P\left(a_{n}\left(M_{n}-b_{n}\right) \leq x\right)=G(x)$ (up to location $G(x) \rightarrow G(x+b)$ and scale $G(x) \rightarrow G(a x), a>0$, changes). These distributions are called extreme type distributions, Type I, II or III [22]. We say a stationary process $\left\{X_{n}\right\}$ satisfies the law of types if, when under linear scaling, the successive maxima $\left\{M_{n}\right\}$ converge to a non-degenerate distribution, then the distribution is a Type I, II or III distribution. We recall the form of these extremal distributions: Type I

$$
G(x)=e^{-e^{-x}}, \quad-\infty<x<\infty .
$$

\section{Type II}

$$
G(x)= \begin{cases}0 & \text { if } x \leq 0 \\ e^{-x^{-\alpha}} & \text { for some } \alpha>0 \text { if } x>0 .\end{cases}
$$

Type III

$$
G(x)= \begin{cases}e^{-(-x)^{\alpha}} & \text { for some } \alpha>0 \text { if } x \leq 0 \\ 1 & \text { if } x>0\end{cases}
$$

If $\left\{X_{n}\right\}$ is a stationary sequence we let $\left\{\hat{X}_{n}\right\}$ denote the associated stationary, independent sequence, that is $\left\{\hat{X}_{n}\right\}$ is independent and $\hat{X}_{1}$ has the same distribution as $X_{1}$. We denote the corresponding derived sequence of maxima for $\left\{\hat{X}_{n}\right\}$ by $\left\{\hat{M}_{n}\right\}$. For $v \in \mathbb{R}$ and sequences $a_{n}, b_{n}$ we define $u_{n}(v)=\frac{v}{a_{n}}+b_{n}$ so that $P\left(M_{n} \leq u_{n}(v)\right)=P\left(a_{n}\left(M_{n}-b_{n}\right) \leq v\right)$. For fixed $v$ we will often drop the dependence upon $v$ and write simply the sequence $u_{n}$. Leadbetter [22] gives two conditions called $D\left(u_{n}\right)$ and $D^{\prime}\left(u_{n}\right)$ for suitable sequences $u_{n}$ which imply that $P\left(M_{n} \leq u_{n}(v)\right) \rightarrow G(v)$ is

Date: June 16, 2010.

2000 Mathematics Subject Classification. Primary 37A50; Secondary 60G70.

Key words and phrases. Lozi maps, Sinai dispersing billiards, extreme value theory, dynamical systems.

The research of MN was supported in part by the National Science Foundation grants DMS-0607345 and DMS0600927. MN would also like to thank the Mittag-Leffler Institute, Djursholm, Sweden for hospitality and support during the writing of this paper. 
equivalent to $P\left(\hat{M}_{n} \leq u_{n}(v)\right) \rightarrow G(v)$. If $\left\{X_{n}\right\}$ satisfies condition $D\left(u_{n}\right)$ then the stochastic process satisfies the law of types. Moreover it is known that

$$
n P\left(X_{0}>u_{n}\right) \rightarrow \tau
$$

is equivalent to $P\left(M_{n} \leq u_{n}\right) \rightarrow e^{-\tau}$ when $D\left(u_{n}\right)$ and $D^{\prime}\left(u_{n}\right)$ hold, and hence we have a strategy for determining the extreme type distribution for dependent sequences.

There are, however, no general techniques for proving conditions $D\left(u_{n}\right)$ and $D^{\prime}\left(u_{n}\right)$ and the latter is usually hard. Collet [8] in an elegant paper used the rate of decay of correlation of Hölder observations to establish $D\left(u_{n}\right)$ for certain one-dimensional non-uniformly expanding maps. Freitas et al [12], based on Collet's work, in turn gave a condition $D_{2}\left(u_{n}\right)$ which has the full force of $D\left(u_{n}\right)$ in that together with $D^{\prime}\left(u_{n}\right)$ it ensures the equivalence of $P\left(M_{n} \leq u_{n}(v)\right) \rightarrow G(v)$ and $P\left(\hat{M}_{n} \leq u_{n}(v)\right) \rightarrow G(v)$. Condition $D_{2}\left(u_{n}\right)$ is easier to establish in the dynamical setting by estimating the rate of decay of correlations of Hölder continuous observables or those of bounded variation.

We establish condition $D_{2}\left(u_{n}\right)$ in this paper for the time-series of certain observations on maps modeled by Young towers with exponential return time tails satisfying Assumption A (described in Section 1.1), which bounds the measure of the outer shell or annulus of a ball. In particular we extend Collet's approach to handle dynamical systems with stable foliations. We also establish condition $D^{\prime}\left(u_{n}\right)$ for planar dispersing billiard maps and flows, a class of Lozi-maps and a class of Lorenz-like maps and show that from the point of view of extreme value theory they behave as i.i.d. processes. Our results on billiard flows are immediate consequences of the results in [20] which show in essence that suspension flows inherit the extreme value behavior of their base transformations. As in Collet [8] will consider the observation $\phi(x)=-\log d\left(x, x_{0}\right)$ on the metric space $(M, d)$. Knowledge of the extreme value statistics for this observation determines the extreme value statistics of a wide class of observations which are functions of $d\left(x, x_{0}\right)$ and maximized (or minimized) at the point $x_{0}[20]$.

Let $B(x, r)$ denote the ball of radius $r>0$ about $x \in M$. For a measure preserving transformation $T:(M, \mu) \rightarrow(M, \mu)$ define, if it exists,

$$
d(x)=\lim _{r \rightarrow 0} \frac{\log \mu(B(x, r))}{\log (r)}
$$

Ledrappier [23] showed that if $\mu$ is an SRB-measure for a $C^{1+\alpha}$ diffeomorphism then the limit $d(x)$ exists and has the same value for $\mu$ almost every $x$ [3]. We assume the existence of a scaling sequence $u_{n}$ such that $n \mu\left(B\left(x_{0}, e^{-u_{n}}\right)\right) \rightarrow e^{-v}$ for $\mu$ a.e. $x_{0}$ and prove our results with respect to this sequence $u_{n}$. Planar dispersing billards possess an absolutely continuous invariant measure, with density $\rho(x):=\frac{d \mu}{d m}(x)$. By the Lebesgue differentiation theorem for any $a>0, n \mu B\left(x_{0}, \frac{\sqrt{a}}{\sqrt{n}}\right) \rightarrow \rho\left(x_{0}\right) a$ for $\mu$ a.e. $x_{0}$. Similarly the class of Lorenz-like maps we consider possess an absolutely continuous invariant measure with density of bounded variation.

Lozi maps have an SRB measure $\mu$ with absolutely continuous conditional measures on local unstable manifolds. In fact the conditional measure on a local unstable manifold is one-dimensional Lebesgue measure. Hence the $\mu$ measure of an annulus about a generic point $x_{0}$ of inner radius $r$ and width $\epsilon$ is bounded by $C \sqrt{\epsilon}$ and hence for $\mu$ a.e. $x_{0}$, the function $r \rightarrow \mu\left(B\left(x_{0}, r\right)\right.$ is continuous and strictly increasing. Thus we may choose a sequence $u_{n}$ satisfying $n \mu\left(B\left(x_{0}, e^{-u_{n}}\right)\right) \rightarrow e^{-v}$ for $\mu$ a.e. $x_{0}$ in this setting also. An immediate corollary of the existence of the dimension $d$ is that $d u_{n} \approx v+\log n$ (in a sense made precise in Lemma 3.2).

The relation $d u_{n} \approx v+\log n$ does not imply that $\lim _{n \rightarrow \infty} \mu\left(M_{n} \leq u_{n}(v)\right)=\lim _{n \rightarrow \infty} \mu\left(M_{n} \leq\right.$ $(\log n+v) / d)$ but rather that for all $\left.\epsilon>0, \lim _{n \rightarrow \infty} \mu\left(M_{n} \leq(1-\epsilon)(\log n+v) / d\right)\right) \leq G(v) \leq$ $\left.\lim _{n \rightarrow \infty} \mu\left(M_{n} \leq(1+\epsilon)(\log n+v) / d\right)\right)$. In the case of the Lozi map this is the best we can do. For Sinai dispersing billiards, as the invariant measure is absolutely continuous, we are able to 
obtain the scaling constants $u_{n}$ explicitly. We prove that for $\mu$ a.e. $x_{0}$, if $\phi(x)=-\log d\left(x, x_{0}\right)$ then $\lim _{n \rightarrow \infty} \mu\left(M_{n} \leq\left(\log n+v+\log \left(\rho\left(x_{0}\right)\right)\right) / 2\right)=e^{-v}$ where $\rho\left(x_{0}\right):=\frac{d \mu}{d m}\left(x_{0}\right)$.

We now state conditions $D_{2}\left(u_{n}\right)$ and $D^{\prime}\left(u_{n}\right)$. If $\left\{X_{n}\right\}$ is a stochastic process define

$$
M_{j, l}:=\max \left\{X_{j}, X_{j+1}, \ldots, X_{j+l}\right\} .
$$

We will often write $M_{0, n}$ as $M_{n}$.

Condition $D_{2}\left(u_{n}\right)$ [12] We say condition $D_{2}\left(u_{n}\right)$ holds for the sequence $X_{0}, X_{1}, \ldots$, if for any integers $l, t$ and $n$

$$
\left|\mu\left(X_{0}>u_{n}, M_{t, l} \leq u_{n}\right)-\mu\left(X_{0}>u_{n}\right) \mu\left(M_{l} \leq u_{n}\right)\right| \leq \gamma(n, t)
$$

where $\gamma(n, t)$ is non-increasing in $t$ for each $n$ and $n \gamma\left(n, t_{n}\right) \rightarrow 0$ as $n \rightarrow \infty$ for some sequence $t_{n}=o(n), t_{n} \rightarrow \infty$.

Condition $D^{\prime}\left(u_{n}\right)[22]$ We say condition $D^{\prime}\left(u_{n}\right)$ holds for the sequence $X_{0}, X_{1}, \ldots$, if

$$
\lim _{k \rightarrow \infty} \limsup _{n} n \sum_{j=1}^{[n / k]} \mu\left(X_{0}>u_{n}, X_{j}>u_{n}\right)=0 .
$$

Condition $D^{\prime}\left(u_{n}\right)$ as stated is not particularly illuminating but is standard in the literature. We will show more precisely the way the condition is used in our proofs.

Collet [8] demonstrated a technique involving maximal functions for establishing $D^{\prime}\left(u_{n}\right)$ for one dimensional non-uniformly expanding maps modeled by a Young tower. His argument relies on the absence of a stable direction and the boundedness of the derivative and these are obstacles to generalizing his argument. The one-dimensional feature can be generalized to expanding maps in higher dimension [14].

We consider a class of maps of Riemannian manifolds, perhaps with singularities, modeled by a Young tower [35] with SRB measure $\mu$ and exponential return time tails. Lozi-like maps and Sinai dispersing billiards fit into this scheme. As a further application we also consider one-dimensional Lorenz-like maps $[17,10]$. We establish $D_{2}\left(u_{n}\right)$ for the process $X_{n}(x)=-\log \left(d\left(x_{0}, T^{n} x\right)\right)$. The proof of $D_{2}\left(u_{n}\right)$ requires only sufficiently high polynomial decay of correlations but as our applications all have exponential decay of correlations to simplify exposition we assume exponential tails. Furthermore if $D^{\prime}\left(u_{n}\right)$ can be verified for these systems, then the process has the same extreme value statistics as its associated i.i.d. process, even for more general observations [20]. We verify $D^{\prime}\left(u_{n}\right)$ for the systems we mentioned but we do not have a general method to establish $D^{\prime}\left(u_{n}\right)$ for all systems modeled by a Young Tower. Our method of proof for $D^{\prime}\left(u_{n}\right)$ in these cases is an extension of the argument in Collet [8].

We note that Poisson-limit laws for return-time statistics in the Axiom-A setting have been established by Hirata [19] and in the uniformly partially hyperbolic setting by Dolgopyat [11]. For recent related work on extreme value theory for deterministic dynamical systems see $[13,12,14,20]$.

Remark 1.0.1. Our results also have implications for hitting time statistics and return time statistics of billiard maps, Lozi maps and Lorenz-like maps. For a map $T: M \rightarrow M$ with invariant ergodic probability measure $\mu$, we may define hitting and return time statistics as follows. For a set $A \subset M$, let $R_{A}(x)$ denote the first time $j \geq 1$ such that $T^{j}(x) \in A$. Given a sequence of sets $\left\{U_{n}\right\}_{n \in \mathbb{N}}$, with $\mu\left(U_{n}\right) \rightarrow 0$ then we say that the system has hitting time statistics (HTS) with distribution $G(t)$ for $\left\{U_{n}\right\}$ if for all $t \geq 0$

$$
\lim _{n \rightarrow \infty} \mu\left(R_{\mid\left(X>u_{n}\right)} \geq \frac{t}{\mu\left(U_{n}\right)}\right)=G(t) .
$$

In applications often the sequence $U_{n}$ is a nested sequence of balls $B\left(x_{0}, \delta_{n}\right)$ of radius $\delta_{n}$ about a point $x_{0}$. 
We say that the system has HTS $G(t)$ to balls at $x_{0}$ if for any sequence $\delta_{n} \subset \mathbb{R}^{+}$, with $\delta_{n} \rightarrow 0$ as $n \rightarrow \infty$ we have HTS $G(t)$ for $U_{n}=B\left(x_{0}, \delta_{n}\right)$.

Analogously we say that return time statistics (RTS) with distribution $G(t)$ holds for $\left\{U_{n}\right\}$ if we can replace the measure $\mu$ by the conditional measure $\mu$ in equation (2), where $\mu_{A}=\frac{\mu \mid A}{\mu(A)}$. RTS to balls is defined analogously to HTS to balls.

Haydn et al [18] show that given a sequence of sets $\left\{U_{n}\right\}$ with $\mu\left(U_{n}\right) \rightarrow 0$, the distribution function of the normalized hitting times to $U_{n}$ converges weakly to a subprobability distribution $F$ if and only if the distribution function of the normalized return time converges weakly to some distribution function $\tilde{F}$ and that if convergent

$$
F(t)=\int_{0}^{t}(1-\tilde{F}(s)) d s .
$$

This in particular shows that the asymptotics for return times is exponential $\left(G(t)=e^{-t}\right)$ if and only if the one for hitting times is also.

Freitas et al [14, Theorem 2] an equivalence between extreme value laws and hitting time statistics (and hence return time statistics via [18]) was obtained for dynamical systems $(M, T, \mu)$ admitting an absolutely continuous invariant probability measure $\mu$. Our results Theorem 4.1 and Theorem 4.3 show if $\phi$ is maximized at a generic Lebesgue density point then $\left\{M_{n}\right\}$ behaves from the point of view of extreme value theory as the corresponding i.i.d. sequence $\left\{\tilde{M}_{n}\right\}$. By Freitas et al our results imply HTS and RTS with an exponential law i.e. $G(t)=e^{-t}$ for nested balls about $\mu$ a.e. $x_{0}$ for planar dispersing billiards and Lozi-like maps.

In subsequent work Freitas et al [15] extend [14, Theorem 2] to invariant measures which are not absolutely continuous with respect to volume. In particular they consider observations $\phi(x)=g\left(r_{x_{0}}(x)\right)$ which are functions of $r_{x_{0}}(x)=\mu\left(B\left(x_{0}, d\left(x, x_{0}\right)\right)\right.$ for a distinguished point $x_{0}$ and determine an equivalence between HTS (as well as RTS) and extreme value statistics for observations $g\left(r_{x_{0}}(x)\right)$. In the setting of Lozi maps the function $r_{x_{0}}(x)$ is continuous as a function of $x$ for $\mu$ a.e. $x_{0}$. The proof of Theorem 4.2 extends immediately to the observation $\phi(x)=-\log r_{x_{0}}(x)$ with scaling constants $u_{n}=\log n+v$. Thus Theorem [15, Theorem 4] combined with Theorem 4.2 implies exponential hitting and return time statistics for balls centered on $x_{0}$ for $\mu$ a.e. $x_{0}$ in the setting of Lozi maps. We gave our extreme value results Theorem 4.2 for observations which are functions of $d\left(x, x_{0}\right)$ as these occur more naturally in applications.

1.1. Statement of results. Let $M$ be a Riemannian manifold with Lebesgue measure $m$ and let $T: M \rightarrow M$ be a (local) diffeomorphism modeled by a Young Tower. The Young Tower assumption implies that there exists a subset $\Lambda \subset M$ such that $\Lambda$ has a hyperbolic product structure and that (P1)-(P4) of [35] hold. We refer the reader to Young's paper [35] and the book by Baladi [1] for details. A similar axiomatic construction of a tower is given by Chernov [6] which is a good reference for background on dispersing billiard maps and flows.

By taking $T$ to be a local diffeomorphism we allow the map $T$ or its derivative to have discontinuities or singularities.

We assume,

Assumption A : For $\mu$ a.e. $x_{0} \in M$ there exists $\tilde{d}:=\tilde{d}\left(x_{0}\right)>0$ such that if $A_{r, \epsilon}\left(x_{0}\right)=\{y \in M$ : $\left.r \leq d\left(x_{0}, y\right) \leq r+\epsilon\right\}$ is a shell of inner radius $r$ and outer radius $r+\epsilon$ about the point $x_{0}$ and if $r$ sufficiently small, $0<\epsilon \ll r<1$, then $\mu\left(A_{r, \epsilon}\left(x_{0}\right)\right) \leq \epsilon^{\tilde{d}}$.

For systems modeled by a Young tower with exponential return time tails satisfying Assumption A we will verify condition $D_{2}\left(u_{n}\right)$. Planar dispersing billiards with finite horizon, Lozi-like maps and Lorenz-like maps satisfy Assumption A and may be modeled by a Young Tower with exponential return time tails. For planar dispersing billiards with infinite horizon we will use the results of [6]. For these systems we also verify condition $D^{\prime}\left(u_{n}\right)$. Our method of proof uses ideas from Collet [8] 
but the arguments need to be modified due to the stable foliation, unbounded derivative and, in the case of Lozi maps, the dissipative nature of the SRB measure.

1.2. Framework of the proof. Suppose we have a Young Tower with exponential return time tails for a local diffeomorphism $T: M \rightarrow M$ of a Riemannian manifold $M$ equipped with Lebesgue measure $m$.

We assume that there is a set $\Lambda$ with a hyperbolic product structure as in Young [35]. Define $\Delta_{0}:=\Lambda$. Let $\Lambda_{0, i}$ be a countable partition of $\Delta_{0}$. Let $R: \Delta_{0} \rightarrow \mathbb{N}$ be an $L^{1}(m)$ return time function with the property that

Define the Young Tower by

$$
\left.R\right|_{\Lambda_{0, i}}:=R_{i}
$$

$$
\Delta=\cup_{i, l \leq R_{i}-1}\left\{(x, l): x \in \Lambda_{0, i}\right\}
$$

and the tower map $F: \Delta \rightarrow \Delta$ by

$$
F(x, l)=\left\{\begin{array}{ll}
(x, l+1) & \text { if } x \in \Lambda_{0, i}, l<R_{i}-1 \\
\left(T^{R_{i}} x, 0\right) & \text { if } x \in \Lambda_{0, i}, l=R_{i}-1
\end{array} .\right.
$$

For convenience, we will refer to $\Delta_{0}:=\cup_{i}\left(\Lambda_{0, i}, 0\right)$ as the base of the tower $\Delta$ and denote $\Lambda_{i}:=\Lambda_{0, i}$. We define $\Delta_{l}=\{(x, l): l<R(x)\}$, the lth level of the tower. Define the map $f=T^{R}: \Delta_{0} \rightarrow \Delta_{0}$ i.e. $f(x)=T^{R(x)}(x)$. We may form a quotiented tower (see [35] for details) by introducing an equivalence relation for points on the same stable manifold. Much of the analysis for the statistical properties of the tower in [35] (but not in this paper) is performed on the quotiented tower, we will merely list the features of the Tower that we will use.

There exists an invariant measure $m_{0}$ for $f: \Delta_{0} \rightarrow \Delta_{0}$ which has absolutely continuous conditional measures on local unstable manifolds in $\Delta_{0}$, with density bounded uniformly from above and below.

The tower structure allows us to construct an invariant measure $\nu$ for $F$ on $\Delta$ by defining for a measurable set $B \subset \Lambda_{l}, \nu(B)=\frac{m_{0}\left(F^{-l} B\right)}{\int \Lambda_{0} R d m_{0}}$ and extending the definition to disjoint unions of such sets in the obvious way. We define a projection $\pi: \Delta \rightarrow M$ by $\pi(x, l)=T^{l}(x)$. We note that $\pi \circ F=T \circ \pi$. The invariant measure $\mu$, which is an SRB measure for $T: M \rightarrow M$, is given by $\mu=\pi_{*} \nu . W_{l o c}^{s}(x)$ will denote the local stable manifold through $x$ i.e there exists $\epsilon(x)>0$ and $C>0$, $0<\alpha<1$ such that $W_{l o c}^{s}=\{y: d(x, y)<\epsilon\}$ and $d\left(T^{n} y, T^{n} x\right)<C \alpha^{n}$ for all $n \geq 0$. We use the notation $W_{l o c}^{s}$ rather than $W_{\epsilon}^{s}(x)$ in contexts where the length of the local stable manifold is not important. We analogously define $\left.W_{l o c}^{u}(x)\right)$ and let $B(x, r)$ denote the ball of radius $r$ centered at the point $x$. We lift a function $\phi: M \rightarrow \mathbb{R}$ to $\Delta$ by defining, with abuse of notation, $\phi(x, l)=\phi\left(T^{l} x\right)$.

Henceforth, we will fix a reference point $x_{0}$ in the support of $\mu$ and define a stochastic process $X_{n}$ given by $X_{n}(x)=-\log d\left(T^{n} x, x_{0}\right)$. This observation determines the extreme value distribution of more general functions with unique maximum at the point $x_{0}[20,14]$. We are interested in the distribution of the maximum of $X_{n}$, denoted by

$$
M_{n}=\max \left\{X_{0}, X_{1}, \ldots, X_{n}\right\} .
$$

We will prove the condition $D_{2}\left(u_{n}\right)$ [12] for a sequence $u_{n}$ for which $n \mu\left(B\left(x_{0}, e^{-u_{n}(v)}\right)\right) \rightarrow e^{-v}$ for some $v \in \mathbb{R}$. We define $\kappa(n)$ to be the rate of decay of correlations of Lipschitz functions with respect to the $\mathrm{SRB}$ measure $\mu$ on the manifold: so that

$$
\left|\int_{M} \phi \psi \circ T^{n} d \mu-\int_{M} \phi d \mu \int_{M} \psi d \mu\right| \leq \kappa(n)\|\phi\|_{L i p}\|\psi\|_{L i p}
$$

for all Lipschitz $\phi, \psi: M \rightarrow \mathbb{R}$. In fact we may use the $L^{\infty}$ norm of $\psi$ in the estimate above as $\psi$ is defined on the quotiented tower (see [35, Section 4]) and in general a faster decay rate than $\kappa(n)$. We assume in this paper that there exists $\theta \in(0,1)$ such that $\kappa(n) \leq \theta^{n}$. 
We define

$$
B_{r, k}\left(x_{0}\right)=\left\{x: T^{k}\left(W_{\eta}^{s}(x)\right) \cap \partial B\left(x_{0}, r\right) \neq \emptyset\right\}
$$

where $B\left(x_{0}, r\right)$ is the ball of radius $r>0$ about $x_{0}$.

An immediate consequence of Assumption A is the following:

Proposition 1.1. Under Assumption $A$ there exist constants $C>0$ and $0<\tau_{1}<1$ such that for any $r, k$

$$
\mu\left(B_{r, k}\left(x_{0}\right)\right) \leq C \tau_{1}^{k} .
$$

Proof. As a consequence of $[35,(\mathrm{P} 2)]$, there exist $\alpha \in(0,1)$ and $C>0$ such that $d\left(T^{n}(x), T^{n}(y)\right) \leq$ $C \alpha^{n}$ for all $y \in W_{\eta}^{s}(x)$. In particular, this implies that $\left|T^{k}\left(W_{\eta}^{s}(x)\right)\right| \leq C \alpha^{k}$ where $|\ldots|$ denotes the length with respect to the Lebesgue measure. Therefore, $T^{k}\left(B_{r, k}\left(x_{0}\right)\right)$ lies in an annulus of width $2 C \alpha^{k}$ around the boundary of the ball of radius $r$ centered at the point $x_{0}$. By Assumption $\mathrm{A}$ and invariance of $\mu$ the result follows.

\section{Condition $D_{2}\left(u_{n}\right)$}

In this section, we establish condition $D_{2}\left(u_{n}\right)$ for maps modeled by a Young Tower with exponential tails satisfying Assumption A. Our main theorem for this section is:

Theorem 2.1. Let $T:(M, \mu) \rightarrow(M, \mu)$ be a dynamical system modeled by a Young Tower with exponential tails satisfying Assumption A. Then the stochastic process $X_{n}:=-\log d\left(T^{n} x, x_{0}\right)$ satisfies the condition $D_{2}\left(u_{n}\right)$, namely, for any integers $j, l$ and $n$,

$$
\left|\mu\left(\left\{X_{0}>u_{n}\right\} \cap\left\{M_{j, l} \leq u_{n}\right\}\right)-\mu\left(\left\{X_{0}>u_{n}\right\}\right) \mu\left(\left\{M_{0, l} \leq u_{n}\right\}\right)\right| \leq \gamma(n, j)
$$

where $\gamma(n, j)$ is non-increasing in $j$ for each $n$ and $n \gamma\left(n, t_{n}\right) \rightarrow 0$ as $n \rightarrow \infty$ for some sequence $t_{n}=o(n), t_{n} \rightarrow \infty$.

We now show how $D_{2}\left(u_{n}\right)$ is used, along with a version of $D^{\prime}\left(u_{n}\right)$ to obtain extreme laws. This uses a blocking argument of Collet [8] based on extreme value statistics (Collet attributes this approach to Galambos [16]).

2.1. The Blocking Argument. We will divide successive observations $\left\{X_{0}, \ldots, X_{n-1}\right\}$ of length $n$ into $q$ blocks of length $p+t$. The gap $t$ will be large enough that successive $p$ blocks are approximately independent but small enough so that $\mu\left(M_{n} \leq u_{n}\right)$ is approximately equal to $\mu\left(M_{q(p+t)} \leq u_{n}\right)$. For the purposes of our applications, which have exponential decay of correlations, we may take $p \approx \sqrt{n}$ and $t=t_{n}=(\log n)^{5}$ but the method is quite flexible. Using approximate independence of $p$ blocks we show $\mu\left(M_{n} \leq u_{n}\right) \approx \mu\left(M_{p} \leq u_{n}\right)^{q}$ and $\mu\left(M_{n} \leq u_{n}\right) \approx 1-p \mu\left(M_{p} \geq u_{n}\right)$. More precisely Collet, using general set inclusions and probabilistic arguments shows

$$
\left|\mu\left(M_{n} \leq u_{n}\right)-\left(1-p \mu\left(X_{0}>u_{n}\right)\right)^{q}\right| \leq q \Gamma_{n}
$$

where

$$
\Gamma_{n}=p \gamma(n, t)+t \mu\left(X_{0}>u_{n}\right)+2 p \sum_{j=1}^{p-1} \mu\left(\left\{X_{0}>u_{n}\right\} \cap\left\{X_{j}>u_{n}\right\}\right) .
$$

By assumption

so

$$
\lim _{n \rightarrow \infty} n \mu\left(X_{0}>u_{n}\right)=e^{-v}
$$

$$
\lim _{n \rightarrow \infty} \mu\left(M_{n} \leq u_{n}\right)=e^{-e^{-v}}
$$

provided $q \Gamma_{n} \rightarrow 0$. The term $p q \gamma\left(n, t_{n}\right) \rightarrow 0$ from $D_{2}\left(u_{n}\right)$ while $q t \mu\left(X_{0}>u_{n}\right) \rightarrow 0$ as $n \mu\left(X_{0}>\right.$ $\left.u_{n}\right) \rightarrow e^{-v}$ and $t=o(n)$. Finally we need to check $n \sum_{j=1}^{p-1} \mu\left(\left\{X_{0}>u_{n}\right\} \cap\left\{X_{j}>u_{n}\right\}\right) \rightarrow 0$. This is condition $D^{\prime}\left(u_{n}\right)$. In applications $p$ is prescribed as a function of $n$ (for example $p=\sqrt{n}$ ). In our 
applications we will give more details in our proofs on the interplay of non-recurrence and decay of correlations needed to ensure $n \sum_{j=1}^{p-1} \mu\left(\left\{X_{0}>u_{n}\right\} \cap\left\{X_{j}>u_{n}\right\}\right) \rightarrow 0$.

\section{Proof of Theorem 2.1.}

We now turn to the proof of Theorem 2.1. The constant $\tau_{1}$ below is from Assumption A 1.1.

Lemma 3.1. Suppose $\Phi: M \rightarrow \mathbb{R}$ is Lipschitz and $\Psi_{a, b}$ is the indicator function

$$
\Psi_{a, b}:=1_{\left\{X_{a} \leq u_{n}, X_{a+1} \leq u_{n}, \ldots, X_{a+b} \leq u_{n}\right\}}
$$

Then for all $j \geq 0$

$$
\left|\int \Phi \Psi_{0, l} \circ T^{j} d \mu-\int \Phi d \mu \int \Psi_{0, l} d \mu\right| \leq \mathcal{O}(1)\left(\|\Phi\|_{\infty} \tau_{1}^{\lfloor j / 2\rfloor}+\|\Phi\|_{L i p} \theta^{\lfloor j / 2\rfloor}\right)
$$

Proof. Define the function $\tilde{\Phi}: \Delta \rightarrow \mathbb{R}$ by $\tilde{\Phi}(x, r)=\Phi\left(T^{r}(x)\right)$ and the function $\tilde{\Psi}_{a, b}(x, r)=$ $\Psi_{a, b}\left(T^{r}(x)\right)$. We choose a reference unstable manifold $\tilde{\gamma}^{u} \subset \Delta_{0}$ and by the hyperbolic product structure each local stable manifold $W_{\eta}^{s}(x)$ will intersect $\tilde{\gamma}^{u}$ in a unique point $\hat{x}$. Here $x$ denotes a point in the base of the tower $\Delta_{0}$ and we therefore have $x \in W_{\eta}^{s}(\hat{x})$.

We define the function $\bar{\Psi}_{a, b}(x, r):=\Psi_{a, b}(\hat{x}, r)$. We note that $\bar{\Psi}_{a, b}$ is constant along stable manifolds in $\Delta$ and the set of points where $\bar{\Psi}_{a, b} \neq \tilde{\Psi}_{a, b}$ is, by definition, the set of $(x, r)$ which project to points $T^{r}(x)$ for which there exist $x_{1}, x_{2}$ on the same local stable manifold as $T^{r}(x)$ for which

but

$$
x_{1} \in\left\{X_{a} \leq u_{n}, \ldots, X_{a+b} \leq u_{n}\right\}
$$

$$
x_{2} \notin\left\{X_{a} \leq u_{n}, \ldots, X_{a+b} \leq u_{n}\right\}
$$

This set is contained inside $\cup_{k=a}^{a+b} T^{-k} B_{u_{n}, k}$. If we let $a=\lfloor j / 2\rfloor$ and $b=l$ then by Proposition 1.1 we have

$$
\nu\left\{\tilde{\Psi}_{\lfloor j / 2\rfloor, l} \neq \bar{\Psi}_{\lfloor j / 2\rfloor, l}\right\} \leq \sum_{k=\lfloor j / 2\rfloor}^{l} \mu\left(B_{u_{n}, k}\right) \leq \mathcal{O}(1) \tau_{1}^{\lfloor j / 2\rfloor} .
$$

By the decay of correlations as proved in [35] under the assumption of exponential tails, we have

$$
\left|\int \tilde{\Phi} \bar{\Psi}_{\lfloor j / 2\rfloor, l} \circ F^{j-\lfloor j / 2\rfloor} d \nu-\int \tilde{\Phi} d \nu \int \bar{\Psi}_{\lfloor j / 2, l\rfloor} d \nu\right| \leq \mathcal{O}(1)\|\Phi\|_{\operatorname{Lip}}\|\Psi\|_{\infty} \theta^{\lfloor j / 2\rfloor} .
$$

Therefore,

$$
\begin{array}{r}
\left|\int \Phi \Psi_{\lfloor j / 2\rfloor} \circ T^{j-\lfloor j / 2\rfloor} d \mu-\int \Phi d \nu \int \Psi_{\lfloor j / 2\rfloor, l} d \mu\right| \\
=\left|\int \tilde{\Phi} \tilde{\Psi}_{\lfloor j / 2\rfloor, l} \circ F^{j-\lfloor j / 2\rfloor} d \nu-\int \tilde{\Phi} d \nu \int \tilde{\Psi}_{\lfloor j / 2, l\rfloor} d \nu\right| \\
\leq\left|\int \tilde{\Phi}\left(\tilde{\Psi}_{\lfloor j / 2\rfloor, l}-\bar{\Psi}_{\lfloor j / 2\rfloor, l}\right) \circ F^{j-\lfloor j / 2\rfloor} d \nu\right|+\mathcal{O}(1)\|\Phi\|_{L i p} \theta^{\lfloor j / 2\rfloor} \\
+\left|\int \tilde{\Phi} d \nu \int\left(\bar{\Psi}_{\lfloor j / 2\rfloor, l}-\tilde{\Psi}_{\lfloor j / 2\rfloor, l}\right) \circ F^{j-\lfloor j / 2\rfloor} d \nu\right| \\
\leq \mathcal{O}(1)\left(\|\Phi\|_{\infty} \nu\left\{\bar{\Psi}_{\lfloor j / 2\rfloor, l} \neq \tilde{\Psi}_{\lfloor j / 2\rfloor, l}\right\}+\|\Phi\|_{\text {Lip }} \theta^{\lfloor j / 2\rfloor}\right) \\
\leq \mathcal{O}(1)\left(\|\Phi\|_{\infty} \tau_{1}^{\lfloor j / 2\rfloor}+\|\Phi\|_{\text {Lip }} \theta^{\lfloor j / 2\rfloor}\right) .
\end{array}
$$

We complete the proof by observing that $\int \Psi_{0, l} d \mu=\int \Psi_{\lfloor j / 2\rfloor, l} d \mu$ by the $\mu$ invariance of $T$ and that $\Psi_{\lfloor j / 2\rfloor, l} \circ T^{j-\lfloor j / 2\rfloor}=\Psi_{j, l}=\Psi_{0, l} \circ T^{j}$. 
To prove condition $D_{2}\left(u_{n}\right)$, we will approximate the characteristic function of the set $\left\{X_{0}>u_{n}\right\}$ by a suitable Lipschitz function. This approximation will decrease sharply to zero near the boundary of the set $\left\{X_{0}>u_{n}\right\}$. The bound in Lemma 3.1 involves the Lipschitz norm, therefore, we need to be able to bound the increase in this norm. To this end, we prove our next lemma.

Lemma 3.2. (1) For $\mu$ a.e. $x_{0}$ for every $\epsilon>0$ there exists an $N \in \mathbb{N}$ such that for all $n \geq N$

$$
\frac{1}{d+\epsilon}(v+\log n) \leq u_{n}(v) \leq \frac{1}{d-\epsilon}(v+\log n)
$$

(2) Denote by $S\left(n, x_{0}\right):=A_{\left(e^{-u_{n}}-e^{-u_{n}^{2}}, e^{\left.-u_{n}^{2}\right)}\right.}\left(x_{0}\right)$ the annulus formed by the region between balls of radius $e^{-u_{n}}$ and $e^{-u_{n}}-e^{-u_{n}^{2}}$ about $x_{0}$. There exists a $\delta\left(x_{0}\right) \in(0,1)$ such that for $n$ large enough

$$
\mu\left(S\left(n, x_{0}\right)\right) \leq \mathcal{O}(1)\left(n^{-2 \delta v-\delta \log n}\right) .
$$

Proof. (1) By the definition of $d$, for any $\epsilon>0$ there exists an $N_{1}$ such that for all $n \geq N_{1}$, $\left(e^{-u_{n}}\right)^{(d+\epsilon)} \leq \mu\left(B\left(x, e^{-u_{n}}\right)\right) \leq\left(e^{-u_{n}}\right)^{(d-\epsilon)}$. Since we have assumed $\lim _{n \rightarrow \infty} n \mu\left(B\left(x, e^{-u_{n}}\right)\right) \rightarrow e^{-v}$, we must have $\lim \sup n\left(e^{-u_{n}}\right)^{d+\epsilon} \leq e^{-v}$. Since $e^{-v}>0$, this implies given $\eta>0$ there exists $N_{2}$ such that $n\left(e^{-u_{n}}\right)^{d+\epsilon} \leq(1+\eta) e^{-v}$ for all $n \geq N_{2}$.

For the other direction, since $\lim \inf n\left(e^{-u_{n}}\right)^{d-\epsilon} \geq e^{-v}$ there exists $N_{3}$ such that for all $n \geq N_{3}$, $n\left(e^{-u_{n}}\right)^{d-\epsilon} \geq(1-\eta) e^{-v}$. Since $\eta$ was arbitrary the result follows.

(2) The proof follows from part (1) and Assumption A. There exists a $\delta \in(0,1)$ such that

$$
\mu\left(S\left(n, x_{0}\right)\right) \leq \mathcal{O}(1)\left|S\left(n, x_{0}\right)\right|^{\delta}
$$

where $|\cdot|$ denotes the width of the annulus. From part (1),

$$
\begin{aligned}
\left|S\left(x_{0}, n\right)\right|^{\delta} & \leq e^{-\left(u_{n}^{2}\right) \delta} \\
& \leq \exp \left(-\frac{\delta}{(d+\epsilon)^{2}}(v+\log n)^{2}\right) \\
& \leq \mathcal{O}(1)\left(n^{-2 \delta^{\prime} v-\delta^{\prime} \log n}\right)
\end{aligned}
$$

for some $\delta^{\prime}>0$.

We note that if the map $T$ preserves an absolutely continuous measure, as in the case of dispersing billiards, then this estimate can be obtained trivially. We are now ready to prove Theorem 2.1.

Proof of Theorem 2.1. We approximate the indicator function $1_{\left\{X_{0}>u_{n}\right\}}$ by a Lipschitz continuous function $\Phi$ as follows. The set $\left\{X_{0}>u_{n}\right\}$ corresponds to a ball of radius $e^{-u_{n}}$ centered at the point $x_{0}$. We define $\Phi$ to be 1 inside a ball centered at $x_{0}$ of radius $e^{-u_{n}}-e^{-u_{n}^{2}}$ and decaying to 0 at a linear rate on $S\left(n, x_{0}\right)$ so that on the boundary of $\left\{X_{0}>u_{n}\right\}, \Phi$ vanishes. The Lipschitz norm of $\Phi$ is seen to be bounded by $\exp \left(u_{n}^{2}\right)$. Since

$$
\begin{array}{r}
\left|\int 1_{\left\{X_{0}>u_{n}\right\}} \Psi_{\lfloor j / 2\rfloor, l} \circ T^{j-\lfloor j / 2\rfloor} d \mu-\mu\left(X_{0}>u_{n}\right) \int \Psi_{\lfloor j / 2\rfloor, l} d \mu\right| \\
\leq\left|\int\left(1_{\left\{X_{0}>u_{n}\right\}}-\Phi\right) \Psi_{\lfloor j / 2\rfloor, l} d \mu\right| \\
+\mathcal{O}(1)\left(\|\Phi\|_{\infty} j^{2} \tau_{1}^{\lfloor j / 4\rfloor}+\|\Phi\|_{L i p} \theta^{\lfloor j / 2\rfloor}\right) \\
+\left|\int\left(1_{\left\{X_{0}>u_{n}\right\}}-\Phi\right) d \mu \int \Psi_{\lfloor j / 2\rfloor, l} d \mu\right|,
\end{array}
$$


and because $\left\|1_{\left\{X_{0}>u_{n}\right\}}-\Phi\right\|_{1} \leq \mu\left(S\left(n, x_{0}\right)\right.$, we have

$$
\mid \mu\left(\left\{X_{0}>u_{n}\right\} \mid \cap\left\{M_{j, l} \leq u_{n}\right\}\right)-\mu\left(\left\{x_{0}>u_{n}\right\}\right) \mu\left(\left\{M_{0, l} \leq u_{n}\right\}\right) \leq \gamma(n, j)
$$

where

$$
\gamma(n, j)=\mathcal{O}(1)\left(n^{-2 \delta^{\prime} v-\delta^{\prime} \log n}+n^{2 v+\log n} \theta_{1}^{\lfloor j / 2\rfloor}\right)
$$

where $\theta_{1}=\max \left\{\tau_{1}, \theta\right\}$. Let $j=t_{n}=(\log n)^{5}$. Then $n \gamma\left(n, t_{n}\right) \rightarrow 0$ as $n \rightarrow \infty$. Note that we had considerable freedom of choice of $t_{n}$, anticipating our applications we choose $t_{n}=(\log n)^{5}$.

\section{Applications}

In this section we prove condition $D^{\prime}\left(u_{n}\right)$ for some concrete examples. We consider Lozi maps and Sinai dispersing billiards. These are (almost) hyperbolic systems that admit invariant cone fields, but the derivative map $D T$ is discontinuous or singular.

The proofs in these examples differ due to the particular characteristics of these systems, however, we identify for the the reader the main steps in the arguments. We start with

Step 1: Control the measure of the set of points which return rapidly to a neighborhood of themselves. In what follows, the set of points which return to a $1 / k$ neighborhood of themselves in fewer than $(\log k)^{5}$ steps is denoted by $E_{k}$;

Step 2: Use estimates from Step 1 to control the measure of the set of points which return to a neighborhood of the distinguished point $x_{0}$ very quickly (for this we resort to a careful extension of the methods used in the one-dimensional case studied in [8], and in case of the Lozi maps, we require some quantitative estimates on the distortion of lengths by holonomies, see Proposition 4.1 and [9]). In what follows, $F_{k}$ denotes the set of points whose neighborhoods have large overlaps with the sets $E_{k}$. Using the Borel-Cantelli lemma, we show that almost every point does not belong in $F_{k}$ infinitely often;

Step 3: Use a decay of correlations to control the measures of exceedences for iterates between $t_{n}:=(\log n)^{5}$ (see equation (1)) and $p:=\sqrt{n}$ (see Section 2.1).

4.1. Planar Dispersing Billiard Maps and Flows. Let $\Gamma=\left\{\Gamma_{i}, i=1: k\right\}$ be a family of pairwise disjoint, simply connected $C^{3}$ curves with strictly positive curvature on the two-dimensional torus $\mathbb{T}^{2}$. The billiard flow $B_{t}$ is the dynamical system generated by the motion of a point particle in $Q=\mathbb{T}^{2} /\left(\cup_{i=1}^{k}\right.$ ( interior $\left.\Gamma_{i}\right)$ with constant unit velocity inside $Q$ and with elastic reflections at $\partial Q=\cup_{i=1}^{k} \Gamma_{i}$, where elastic means "angle of incidence equals angle of reflection". If each $\Gamma_{i}$ is a circle then this system is called a periodic Lorentz gas, a well-studied model in physics. The billiard flow is Hamiltonian and preserves a probability measure (which is Liouville measure) $\tilde{\mu}$ given by $d \tilde{\mu}=C_{Q} d q d t$ where $C_{Q}$ is a normalizing constant and $q \in Q, t \in \mathbb{R}$ are Euclidean coordinates.

We first consider the billiard map $T: \partial Q \rightarrow \partial Q$. Let $r$ be a one-dimensional coordinatization of $\Gamma$ corresponding to length and let $n(r)$ be the outward normal to $\Gamma$ at the point $r$. For each $r \in \Gamma$ we consider the tangent space at $r$ consisting of unit vectors $v$ such that $(n(r), v) \geq 0$. We identify each such unit vector $v$ with an angle $\theta \in[-\pi / 2, \pi / 2]$. The boundary $M$ is then parametrized by $M:=\partial Q=\Gamma \times[-\pi / 2, \pi / 2]$ so that $M$ consists of the points $(r, \theta) . T: M \rightarrow M$ is the Poincaré map that gives the position and angle $T(r, \theta)=\left(r_{1}, \theta_{1}\right)$ after a point $(r, \theta)$ flows under $B_{t}$ and collides again with $M$, according to the rule angle of incidence equals angle of reflection. Thus if $(r, \theta)$ is the time of flight before collision $T(r, \theta)=B_{h(r, \theta)}(r, \theta)$. The billiard map preserves a measure $d \mu=c_{M} \cos \theta d r d \theta$ equivalent to 2-dimensional Lebesgue measure $d m=d r d \theta$ with density $\rho(x)$ where $x=(r, \theta)$.

Under the assumption of finite horizon condition, namely, that the time of flight $h(r, \theta)$ is bounded above, Young [35] proved that the billiard map has exponential decay of correlations for Hölder observations. This settled a long-standing question about the rate of decay of correlations in such systems. Chernov [6] extended this result to planar dispersing billiards with infinite horizon 
where $h(x, r)<\infty$ for all but finitely many points $(r, \theta)$ but is not essentially bounded. Chernov also proved exponential decay for dispersing billiards with corner points (a class of billiards we do not discuss in this paper). A good reference for background results for this section are the papers $[4,5,35,6]$. We first establish extreme value statistics for billiard maps and then, in the next section, deduce corresponding limit laws for billiard flows using the results of Holland et al [20].

We prove,

Theorem 4.1. Let $T: M \rightarrow M$ be a planar dispersing billiard map. Then for $\mu$ a.e. $x_{0}$ the stochastic process defined by $X_{n}(x)=-\log \left(d\left(x_{0}, T^{n} x\right)\right)$ satisfies a Type I extreme value law in the sense that $\lim _{n \rightarrow \infty} \mu\left(M_{n} \leq\left(v+\log n+\log \left(\rho\left(x_{0}\right)\right)\right) / 2\right)=e^{-e^{-v}}$.

Proof. Assumption A is satisfied by planar dispersing billiards with finite and infinite horizon as the invariant measure is equivalent to Lebesgue. This is proved in [5, Appendix 2] where it is shown that $\tilde{d}$ may be taken as 1 in the case of finite horizon and $4 / 5$ in the case of infinite horizon. The proof of $D_{2}\left(u_{n}\right)$ is immediate in the case of dispersing billiard maps with finite horizon, as they are modeled by a Young Tower in [35], have exponential decay of correlations. Chernov [6, Section 5] (see also [5, Section 5]) constructs a Young Tower for billiards with infinite horizon to prove exponential decay of correlations so that condition $D_{2}\left(u_{n}\right)$ is satisfied by this class of billiard map as well. Hence we need only prove condition $D^{\prime}\left(u_{n}\right)$.

It is known (see [6, Lemma 7.1] for finite horizon and [6, Section 8] for infinite horizon) that dispersing billiard maps expand in the unstable direction in the Euclidean metric $|\cdot|=\sqrt{(d r)^{2}+(d \phi)^{2}}$ , in that $\left|D T_{u}^{n} v\right| \geq C \tilde{\lambda}^{n}|v|$ for some constants $C, \tilde{\lambda}>1$ which is independent of $v$. In fact $\left|L_{n}\right| \geq C \tilde{\lambda}^{n}\left|L_{0}\right|$ where $L_{0}$ is a segment of unstable manifold (once again in the Euclidean metric) and $L_{n}$ is $T^{n} L_{0}$.

We choose $N_{0}$ so that $\lambda:=C \tilde{\lambda}^{N_{0}}>1$ and then $T^{N_{0}}$ (or $D T^{N_{0}}$ ) expands unstable manifolds (tangent vectors to unstable manifolds) uniformly in the Euclidean metric.

It is common to use the $p$-metric in proving ergodic properties of billiards. Young uses this semi-metric in [35]. Recall that for any curve $\gamma$, the $p$-norm of a tangent vector to $\gamma$ is given as $|v|_{p}=\cos \phi(r)|d r|$ where $\gamma$ is parametrized in the $(r, \phi)$ plane as $(r, \phi(r))$. The Euclidean metric in the $(r, \phi)$ plane is given by $d s^{2}=d r^{2}+d \phi^{2}$; this implies that $|v|_{p} \leq \cos \phi(r) d s \leq d s=|v|$. We will use $l_{p}(C)$ to denote the length of a curve in the $p$-metric and $l(C)$ to denote length in the Euclidean metric. If $\gamma$ is a local unstable manifold or local stable manifold then $C_{1} l(\gamma)_{p} \leq l(\gamma) \leq C_{2} \sqrt{l_{p}(\gamma)}$.

For planar dispersing billiards there exists an invariant measure $\mu$ (which is equivalent to 2dimensional Lebesgue measure) and through $\mu$ a.e. point $x$ there exists a local stable manifold $W_{l o c}^{s}(x)$ and a local unstable manifold $W_{l o c}^{u}(x)$. The SRB measure $\mu$ has absolutely continuous (with respect to Lebesgue measure ) conditional measures $\mu_{x}$ on each $W_{l o c}^{u}(x)$. The expansion by $D T$ is unbounded however in the $p$-metric at $\cos \theta=0$ and this may lead to quite different expansion rates at different points on $W_{l o c}^{u}(x)$. To overcome this effect and obtain uniform estimates on the densities of conditional SRB measure it is common to definite homogeneous local unstable and local stable manifolds. This is the approach adopted in [4, 5, 6, 35]. Fix a large $k_{0}$ and define for $k>k_{0}$

$$
\begin{gathered}
I_{k}=\left\{(r, \theta): \frac{\pi}{2}-k^{-2}<\theta<\frac{\pi}{2}-(k+1)^{-2}\right\} \\
I_{-k}=\left\{(r, \theta):-\frac{\pi}{2}+(k+1)^{-2}<\theta<-\frac{\pi}{2}+k^{-2}\right\}
\end{gathered}
$$

and

$$
I_{k_{0}}=\left\{(r, \theta):-\frac{\pi}{2}+k_{0}^{-2}<\theta<\frac{\pi}{2}-k_{0}^{-2}\right\} .
$$

In our setting we call a local unstable (stable) manifold $W_{l o c}^{u}(x),\left(W_{l o c}^{s}(x)\right)$ homogeneous if for all $n \geq 0 T^{n} W_{l o c}^{u}(x)\left(T^{-n} W_{l o c}^{s}(x)\right)$ does not intersect any of the line segments in $\cup_{k>k_{0}}\left(I_{k} \cup I_{-k}\right) \cup I_{k_{0}}$. Homogeneous $W_{l o c}^{u}(x)$ have almost constant conditional SRB densities $\frac{d \mu_{x}}{d m_{x}}$ in the sense that there 
exists $C>0$ such that $\frac{1}{C} \leq \frac{d \mu_{x}\left(z_{1}\right)}{d m_{x}} / \frac{d \mu_{x}\left(z_{2}\right)}{d m_{x}} \leq C$ for all $z_{1}, z_{2} \in W_{l o c}^{u}(x)$ (see [6, Section 2] and the remarks following Theorem 3.1).

From this point on all the local unstable (stable) manifolds that we consider will be homogeneous. Bunimovith et al [5, Appendix 2, Equation A2.1] give quantitative estimates on the length of homogeneous $W_{l o c}^{u}(x)$. They show there exists $C, \tau>0$ such that $\mu\left\{x: l\left(W_{l o c}^{s}(x)\right)<\epsilon\right.$ or $l\left(W_{l o c}^{u}(x)\right)<$ $\epsilon\} \leq C \epsilon^{\tau}$ where $l(C)$ denotes 1-dimensional Lebesgue measure or length of a rectifiable curve $C$. In our setting $\tau$ could be taken to be $\frac{2}{9}$, its exact value will play no role but for simplicity in the forthcoming estimates we assume $0<\tau<\frac{1}{2}$.

The natural measure $\mu$ has absolutely continuous conditional measures $\mu_{x}$ on local unstable manifolds $W_{l o c}^{u}(x)$ which have almost uniform densities with respect to Lebesgue measure on $W_{l o c}^{u}(x)$ by [6, Equation 2.4].

4.1.1. Controlling the measure of the set of rapidly returning points. Let $A_{\sqrt{\epsilon}}=\left\{x:\left|W_{l o c}^{u}(x)\right|>\right.$ $\sqrt{\epsilon}\}$ then $\mu\left(A_{\sqrt{\epsilon}}^{c}\right)<C \epsilon^{\tau / 2}$. Let $x \in A_{\sqrt{\epsilon}}$ and consider $W_{l o c}^{u}(x)$. Since $\left|T^{-k} W_{l o c}^{u}(x)\right|<\lambda^{-1}\left|W_{l o c}^{u}(x)\right|$ for $k>N_{0}$ the optimal way for points $T^{-k}(y)$ in $T^{-k} W_{l o c}^{u}(x)$ to be close to their preimages $y \in W_{l o c}^{u}(x)$ is for $T^{-k} W_{l o c}^{u}(x)$ to overlay $W_{l o c}^{u}(x)$, in which case it has a fixed point and it is easy to see $l\left\{y \in W_{l o c}^{u}(x): d\left(y, T^{-k} y\right)<\epsilon\right\} \leq l\left\{y \in \mathbb{R}: d\left(y, \frac{y}{\lambda}\right)<\epsilon\right\} \leq\left(1-\lambda^{-1}\right) \epsilon$. Accordingly $l\left\{y \in W_{l o c}^{u}(x): d\left(y, T^{-k} y\right)<\epsilon\right\} \leq C \sqrt{\epsilon} l\left\{y \in W_{l o c}^{u}(x)\right\}$. Recalling that the density of the conditional SRB-measure $\mu_{x}$ is bounded above and below with respect to one-dimensional Lebesgue measure we obtain $\mu_{x}\left(A_{\sqrt{\epsilon}}^{c}\right)<C \sqrt{\epsilon}$. Integrating over all unstable manifolds in $A_{\sqrt{\epsilon}}$ (throwing away the set $\left.\mu\left(A_{\sqrt{\epsilon}}^{c}\right)\right)$ we have $\mu\left\{x: d\left(T^{-k} x, x\right)<\epsilon\right)<C \epsilon^{\tau / 2}$. Since $\mu$ is $T$-invariant $\mu\left\{x: d\left(T^{k} x, x\right)<\epsilon\right\}<$ $C \epsilon^{\tau / 2}$ for $k>N_{0}$. Hence for any iterate $T^{k}, k>N_{0}$

$$
\mathcal{E}_{k}(\epsilon):=\mu\left\{x: d\left(T^{k} x, x\right)<\epsilon\right\}<C \epsilon^{\tau / 2} .
$$

Recall that the scaling constant $u_{n}(v)$ is chosen so that $n \mu\left(B\left(x_{0}, e^{-u_{n}(v)}\right) \rightarrow e^{-v}\right.$, for hyperbolic billiards we take $u_{n}(v)=\frac{1}{2}\left(v+\log n+\log \left(\rho\left(x_{0}\right)\right)\right)$ and shrinking balls of radius roughly $\frac{1}{\sqrt{n}}$ about points. This leads to the use of $\frac{1}{\sqrt{k}}$ in the next definition. Define

$$
E_{k}:=\left\{x: d\left(T^{j} x, x\right) \leq \frac{2}{\sqrt{k}} \text { for some } 1 \leq j \leq(\log k)^{5}\right\} .
$$

We have shown that for any $\delta>0$, for all sufficiently large $k, \mu\left(E_{k}\right) \leq k^{-\tau / 4+\delta}$. For simplicity we take $\mu\left(E_{k}\right) \leq k^{-\sigma}$ where $\sigma<\tau / 4-\delta$ and omit the constant $e^{-v}$ in the following equations.

4.1.2. Controlling the measure of the set of points whose neighborhoods have large overlaps with the sets $E_{k}$. Define the Hardy-Littlewood maximal function $M_{l}$ for $\phi(x)=1_{E_{l}}(x) \rho(x)$ where $\rho(x)=$ $\frac{d \mu}{d m}(x)$, so that

$$
M_{l}(x):=\sup _{a>0} \frac{1}{m\left(B_{a}(x)\right)} \int_{B_{a}(x)} 1_{E_{l}}(y) \rho(y) d m(y) .
$$

A theorem of Hardy and Littlewood [27, Theorem 2.19] implies that

$$
m\left(\left|M_{l}\right|>C\right) \leq \frac{\left\|1_{E_{l}} \rho\right\|_{1}}{C}
$$

where $\|,\|_{1}$ is the $L^{1}$ norm with respect to $m$. Let

$$
F_{k}:=\left\{x: \mu\left(B_{k^{-\gamma / 2}}(x) \cap E_{k^{\gamma / 2}}\right) \geq\left(k^{-\gamma \beta / 2}\right) k^{\gamma / 2}\right.
$$

Then $F_{k} \subset\left\{M_{k^{\gamma / 2}}>k^{-\gamma \beta / 2}\right\}$ and hence

$$
m\left(F_{k}\right) \leq \mu\left(E_{k^{\gamma / 2}}\right) k^{\gamma \beta / 2} \leq C k^{-\gamma \sigma} k^{\gamma \beta / 2} .
$$


If we take $0<\beta<\sigma$ and $\gamma>\sigma / 2$ then for some $\delta>0, k^{-\gamma \sigma} k^{\gamma \beta / 2}<k^{-1-\delta}$ and hence

$$
\sum_{k} m\left(F_{k}\right)<\infty
$$

Thus for $m$ a.e. (hence $\mu$ a.e.) $x_{0} \in X$ there exists $N\left(x_{0}\right)$ such that $x_{0} \notin F_{k}$ for all $k>N\left(x_{0}\right)$. Thus along the subsequence $n_{k}=k^{-\gamma / 2}, \mu\left(X_{0}>u_{n_{k}}, X_{0} \circ T^{j}>u_{n_{k}}\right) \leq n_{k}^{-1-\delta}$ for $k>N\left(x_{0}\right)$. This is sufficient to obtain an estimate for all $u_{n}$. Since $\lim _{k \rightarrow \infty}\left(\frac{k+1}{k}\right)^{\gamma / 2}=1$ if $k^{\gamma / 2} \leq n \leq(k+1)^{\gamma / 2}$ then for sufficiently large $n \mu\left(X_{0}>u_{n}, X_{0} \circ T^{j}>u_{n}\right) \leq \mu\left(X_{0}>u_{n_{k}}, X_{0} \circ T^{j}>u_{n_{k}}\right) \leq n_{k}^{-1-\delta} \leq 2 n^{-1-\delta}$.

We now control the iterates $1 \leq j \leq N_{0}$. If $x_{0}$ is not periodic then $\min _{1 \leq i<j \leq N_{0}} d\left(T^{i} x_{0}, T^{j} x_{0}\right) \geq$ $s\left(x_{0}\right)>0$ and hence for large enough $n$, for all $1 \leq j \leq N_{0}, \mu\left(X_{0}>u_{n}, X_{0} \circ T^{j}>u_{n}\right)=0$.

Recalling that $u_{n}$ was chosen so that $n \mu\left(B_{e^{-u_{n}}}(x)\right) \rightarrow e^{-v}$, we get, for any $1 \leq j \leq(\log n)^{5}$,

$$
\mu\left(X_{0}>u_{n}, X_{0} \circ T^{j}>u_{n}\right) \leq 2 n^{-1-\delta} .
$$

Hence

$$
\lim _{n \rightarrow \infty} n \sum_{j=1}^{(\log n)^{5}} \mu\left(X_{0}>u_{n}, X_{0} \circ T^{j}>u_{n}\right)=0 .
$$

4.1.3. Accounting for exceedences between $(\log n)^{5}$ and $\sqrt{n}$. We now use exponential decay of correlations to show

$$
\lim _{n \rightarrow \infty} n \sum_{(\log n)^{5}}^{p=\sqrt{n}} \mu\left(X_{0}>u_{n}, X_{0} \circ T^{j}>u_{n}\right)=0 .
$$

We let $1_{u_{n}}$ denote the indicator function of the set $\left\{X_{0}>u_{n}\right\}$. We approximate the indicator function $1_{u_{n}}$ by a Lipschitz function $\phi_{n}$ which is 1 on a neighborhood of $x_{0}$ of radius $e^{-u_{n}}-e^{-u_{n}^{2}}$ and linearly decaying to 0 on the complement of the ball of radius $e^{-u_{n}}$. $\phi_{n}$ has Lipschitz norm bounded by $e^{\left(u_{n}\right)^{2}} \approx e^{(v+\log n)^{2} / 4}$.

Note that

$$
\begin{aligned}
\left|\int 1_{u_{n}}\left(1_{u_{n}} \circ T^{j}\right) d \mu-\left(\int 1_{u_{n}} d \mu\right)^{2}\right| & \leq\left|\int \phi_{n}\left(\phi_{n} \circ T^{j}\right) d \mu-\left(\int \phi_{n} d \mu\right)^{2}\right| \\
& +\left|\left(\int \phi_{n} d \mu\right)^{2}-\left(\int 1_{u_{n}} d \mu\right)^{2}\right| \\
& +\left|\int 1_{u_{n}}\left(1_{u_{n}} \circ T^{j}\right) d \mu-\int \phi_{n}\left(\phi_{n} \circ T^{j}\right) d \mu\right| .
\end{aligned}
$$

If $(\log n)^{5} \leq j \leq p=\sqrt{n}$ then by decay of correlations $\left|\int \phi_{n}\left(\phi_{n} \circ T^{j}\right) d \mu-\left(\int \phi_{n} d \mu\right)^{2}\right| \leq$ $C e^{2 u_{n}^{2}} \theta^{j} \leq C e^{-2 \log n}=\frac{C}{n^{2}}$ If $n$ is sufficiently large. Furthermore if $n$ is large $\mid\left(\int \phi_{n} d \mu\right)^{2}-$ $\left(\int 1_{u_{n}} d \mu\right)^{2} \mid<C n^{-2(\delta v-\delta \log n)}<C n^{-2}$.

Finally $\left|\int \phi_{n}\left(\phi_{n} \circ T^{j}\right) d \mu-\int 1_{u_{n}}\left(1_{u_{n}} \circ T^{j}\right) d \mu\right| \leq \mu\left(\phi_{n}(x) \neq 1_{\left\{X_{0}>u_{n}\right\}}\right)+\mu\left(\phi_{n} \circ T^{j}(x) \neq\right.$ $\left.1_{\left\{X_{0} \circ T^{j}(x)>u_{n}\right\}}\right) \leq \frac{C}{n^{2}}$.

Hence

$$
\lim _{n \rightarrow \infty} n \sum_{j=(\log n)^{5}}^{p=\sqrt{n}} \mu\left(X_{0}>u_{n}, X_{0} \circ T^{j}>u_{n}\right)=0 .
$$

This concludes the proof of Theorem 4.1. 
4.1.4. Billiard flows. The billiard flow $B_{t}: Q \rightarrow Q$ may be viewed as suspension flow over the billiard map $T: M \rightarrow M$. In Holland et al [20] extreme value laws are derived for suspension flows as a consequence of extreme value laws for base maps.

Suppose $B_{t}: Q \rightarrow Q$ is the billiard flow preserving the ergodic invariant natural measure $\tilde{\mu}$ and $\bar{h}$ is the average first return time of the billiard flow from the boundary to the boundary with respect to $\mu$ i.e. $\bar{h}=C_{M} \int_{M} h(r, \phi) \cos \phi d \phi d r$ where $h(r, \phi)$ is the time of flow till the point $(r, \phi) \in \partial Q$ hits the boundary again $\partial Q$. As a consequence of [20, Theorem 2.10], we have the following corollary,

Corollary 4.1. for $\tilde{\mu}$ a.e. $p_{0} \in Q$ if $\phi(p)=-\log d\left(p, p_{0}\right)$ and $M_{t}:=\max _{s \leq t}\left\{\phi\left(B_{s}(p)\right)\right.$ then

$$
\left.\lim _{t \rightarrow \infty} \tilde{\mu}\left(M_{t} \leq v+\log (t / \bar{h})+\log \rho\left(x_{0}\right)\right) / 2\right)=e^{-e^{-v}} .
$$

4.2. Lozi-like maps. The Lozi mapping $T$ is a homeomorphism of $\mathbb{R}^{2}$ given by

$$
(x, y) \rightarrow(1+y-a|x|, b x)
$$

where $a$ and $b$ are parameters. It has been studied as a model of chaotic dynamics intermediate in complexity (or difficulty) between Axiom A diffeomorphisms and Henon diffeomorphisms [29, 9, 34]. The derivative is discontinuous on the $y$-axis and this leads to arbitrarily short smooth local unstable manifolds. Misuiurewicz [29] proved that there exists an open set $G$ of parameters such that if $(a, b) \in G$ the map $T$ is hyperbolic. If $(a, b) \in G$, then $T_{a, b}$ has invariant stable and unstable directions (where the derivative is defined) and the angle between them is bounded below by $\pi / 5$. We will restrict our attention to maps with parameters in the set $G$.

These maps admit a strict cone, and the tangent derivatives, where defined, satisfy uniform expansion estimates [9] in that there exists $\lambda>1$ such that $\left|D T^{n} v\right| \geq \lambda^{n}|v|$ for all $v \in E^{u}$ (the unstable direction) and correspondingly for $E^{s}$ (the stable direction). $T_{a, b}$ has an invariant ergodic probability measure $\mu[9]$ which is absolutely continuous with respect to the one-dimensional Lebesgue measure along local unstable curves. In fact the conditional invariant measure on local unstable manifolds is simply 1-dimensional Lebesgue measure [9]. Young [34] established similar results for a broader class of maps, 'generalized' Lozi maps which are piecewise $C^{2}$ mappings of the plane. But one reason for restricting to maps $T_{a, b},(a, b) \in G$ is that for such maps Collet and Levy have also shown that for $\mu$ almost every point on the attractor the Hausdorff dimension of $\mu$ exists and is constant [9].

The existence of a dimension $d$ implies that for almost every $x$ in the attractor, the dimension constant $d(x)$ in the definition of $u_{n}$ is the same. We will use a sequence of scaling constants $u_{n}\left(x_{0}, v\right)$ defined for a generic point $x_{0}$ by the requirement that $n \mu\left(B\left(x_{0}, e^{-u_{n}\left(x_{0}, v\right)}\right) \rightarrow e^{-v}\right.$.

In later work [35, Section 7] Young constructs SRB measures via a Tower construction for a broader class of piecewise $C^{2}$ uniformly hyperbolic maps of the plane. The Lozi map $T_{a, b}$ with $(a, b) \in G, b$ sufficiently small may be modeled by a Young Tower with exponential tails [35]. Hence the Lozi maps we consider satisfy exponential decay of correlations for Hölder continuous observations.

We now summarize the ergodic properties of the Lozi maps that we will use. $T$ has an invariant SRB-measure $\mu$ and $\mu$ a.e. point $x$ has a local stable manifold $W_{l o c}^{s}(x)$ and local unstable manifold $W_{l o c}^{u}(x)$. In [9, Proposition IV.1] it is shown that the conditional expectations of $\mu$ on the local unstable manifolds are the corresponding 1-d Lebesgue measures. Furthermore $\mu$ a.e. point $x$ has a quadrilateral $\beta(x)$ with a local product structure, in the sense that $y \in \beta(x)$ implies there exists a unique $z \in \beta(x)$ such that $z=W_{l o c}^{u}(y) \cap W_{l o c}^{s}(x)$ and a unique $z^{\prime} \in \beta(x)$ such that $z^{\prime}=W_{l o c}^{s}(y) \cap$ $W_{l o c}^{u}(x)\left[9\right.$, Section 4]. Suppose that $W_{l o c}^{u}(x)$ and $W_{l o c}^{u}\left(x^{\prime}\right)$ are local unstable manifolds. Then the holonomy $h: W_{l o c}^{u}(x) \rightarrow W_{l o c}^{u}\left(x^{\prime}\right)$ is defined on the set $D(h):=x \in W_{l o c}^{u}(x): W_{l o c}^{s}(x) \cap W_{l o c}^{s}\left(x^{\prime}\right) \neq \emptyset$. The holonomy between local unstable manifolds satisfies the following quantitative estimates, 
Proposition 4.1. [9, Proposition II.4] Given $W_{l o c}^{u}(x)$ and $W_{l o c}^{u}\left(x^{\prime}\right)$ there is a constant $L$ such that for any Borel subset $A \subset W_{l o c}^{u}(x) \cap D(h)$,

$$
\left(1-L\left(d\left(W_{l o c}^{u}(x), W_{l o c}^{u}\left(x^{\prime}\right)\right)^{1 / 3}\right) l(A) \leq l(h(A)) \leq\left(1+L\left(d\left(W_{l o c}^{u}(x), W_{l o c}^{u}\left(x^{\prime}\right)\right)^{1 / 3}\right) l(A) .\right.\right.
$$

Note that the local unstable manifolds lie in a strict cone and the conditional invariant measure on local unstable manifolds is 1-dimensional Lebesgue measure. Suppose that $x_{0} \in M, A_{r, \epsilon}\left(x_{0}\right)$ is an annulus with center $x_{0}$ and $m_{x}$ is conditional measure on $W_{l o c}^{u}(x)$ with $x$ in the quadrilateral $\beta\left(x_{0}\right)$. Since $m_{x}\left(A_{r, \epsilon}\right)<C \sqrt{\epsilon}$ for all $x \in \beta\left(x_{0}\right)$, Assumption A is satisfied. Thus we need only show condition $D^{\prime}\left(u_{n}\right)$. We will establish $D^{\prime}\left(u_{n}\right)$ in this section and prove the following theorem.

Theorem 4.2. Let $T_{a, b}: M \rightarrow M$ be a Lozi map with $(a, b) \in G$ with $b$ sufficiently small. Then for $\mu$ a.e. $x_{0}$ the stochastic process defined by $X_{n}(x)=-\log \left(d\left(x_{0}, T^{n} x\right)\right)$ satisfies a Type I extreme value law in the sense that $\lim _{n \rightarrow \infty} \mu\left(M_{n} \leq u_{n}\left(x_{0}, v\right)\right)=e^{-e^{-v}}$.

Remark 4.2.1. We do not know the precise scaling constants $u_{n}\left(x_{0}, v\right)$, but for all $\epsilon>0$, $\left.\left.\lim _{n \rightarrow \infty} \mu\left(M_{n} \leq(1-\epsilon)(\log n+v) / d\right)\right) \leq e^{-e^{-v}} \leq \lim _{n \rightarrow \infty} \mu\left(M_{n} \leq(1+\epsilon)(\log n+v) / d\right)\right)$ which provides an estimate of the correct sequence $u_{n}$.

Proof. We need only establish $D^{\prime}\left(u_{n}\right)$. We will denote the length of a rectifiable curve $C$ by $l(C)$ in the usual Euclidean metric. As tangent vectors to local unstable manifolds lie in a strict cone the projected length onto either the horizontal or vertical axis of a connected component $\mathcal{C}$ of $T^{k} W_{l o c}^{u}(x)$ is bounded below by $\kappa l(\mathcal{C})$ for some $\kappa>0$. This constant will be absorbed into our $C$ 's below, so that the expansion of a local unstable manifold under $T^{j}$ may be used to estimate the measure of points which satisfy $d\left(x, T^{j} x\right)<\epsilon$. The projection of $W_{l o c}^{u}(x)$ onto the horizontal axis expands uniformly for all $j>N_{0}$ for some $N_{0}$, but as in the case of billiards this does not affect our argument if $x_{0}$ is not a periodic point. For simplicity of exposition we assume $N_{0}=1$.

One would think that as the derivative is bounded and there is uniform expansion in the unstable direction, which lies within a cone, the proof of $D^{\prime}\left(u_{n}\right)$ would be immediate but the presence of discontinuities for the derivative complicates the picture. If $W_{l o c}^{u}(x)$ is a local unstable manifold then $T^{n}\left(W_{l o c}^{u}(x)\right)$ is either a line segment or a connected broken line segment. Here is what could possibly go wrong in the latter case. Suppose that the map $T$ (restricted to local unstable manifolds), expands uniformly and $\left|T^{\prime}(x)\right|>\lambda>1$. Let $L$ be a segment of unstable manifold and consider $T^{n} L$. It expands but may encounter the set of discontinuities/singularities $S$. Suppose $T^{n} L$ is partitioned into $M$ smooth components $\beta_{i}$ with corresponding pre-image intervals $\alpha_{i} \subset L$ so that $T^{n} \alpha_{i}=\beta_{i}$. Suppose the map $T^{n}$ folds back on itself many times and places each $\beta_{i}$ atop $\alpha_{i}$ such that the left endpoint $x_{i}$ of $\alpha_{i}$ very close to the left endpoint $T^{n} x_{i}$ of $\beta_{i}$. If $\left|\beta_{i}\right|<\epsilon$ then each point in $\alpha_{i}$ lies within $\epsilon$ of its image under $T^{n}$. We have to show this cannot happen. We use the structure of a Young Tower to do this.

4.2.1. Controlling the conditional measure of the set of points which return rapidly to a neighborhood (in the phase space) of themselves. We first show there exists $\sigma>0$ such that for a generic point $z$ (generic here means a set of points of full measure) if $\epsilon$ is sufficiently small then $m_{z}\left(y \in W_{l o c}^{u}(z)\right.$ : $\left.d\left(y, T^{j} y\right)<\epsilon\right)<\epsilon^{\sigma} l\left(W_{l o c}^{u}(z)\right)$ where $m_{z}$ is conditional measure on $W_{l o c}^{u}(z)$.

Assume that $z=\pi(x, r)$ for some $(x, r) \in \Delta$ i.e. for some $i, x \in \Lambda_{i} \subset \Lambda_{0}, r<R(x)=R_{i}$ we have $T^{r} x=z$. We may assume without loss of generality that $W_{l o c}^{u}(z) \supset T^{r}\left(W_{l o c}^{u}(x) \cap \Lambda_{i}\right)$, otherwise we could refine the partition on the Tower by defining a new return time on the base $R_{k}(y)=R(y)+\ldots+R\left(f^{k} y\right)$. By refining in this way we could also require $\left(T^{R}\right)^{\prime}(x)>2$. This is equivalent to considering a Tower with return time partition $P_{k}:=\bigvee_{j=0}^{k} f^{-j} P_{0}$ on the base, where $P_{0}$ is the original partition into sets $\left\{\Lambda_{i}\right\}$. For large enough $k, W_{l o c}^{u}(z) \supset T^{r}\left(W_{l o c}^{u}(x) \cap \Lambda_{i}\right)$. Note that the new Tower will also have exponential return time tails. We identify $T^{r}\left(W_{l o c}^{u}(x) \cap \Lambda_{i}\right) \cap W_{l o c}^{u}(z):=$ $W_{\rho}^{u}(x) \subset M$ with $W_{l o c}^{u}(x, r)$ on the Tower. The portion of local unstable manifold $W_{\rho}^{u}(z)$ may not be symmetrical about $z$ but this will not affect our argument. 
There exists $\tau>0$ such that if $\epsilon>0$ is sufficiently small then except for a set of $\Lambda_{i}^{\prime} s$ of $m_{0}$ measure less than $\epsilon^{\tau},\left|W_{\eta}^{u}(y) \cap \Lambda_{i}\right|>\sqrt{\epsilon}$ for all $y:=(y, 0) \in \Lambda_{i}$. This observation uses exponential decay of the return time. To see this suppose that $\Lambda_{n}$ has return time $R_{n}$. Let $\gamma$ be the length of $\Lambda_{0}$ in the unstable direction. Since $\left|T^{\prime}(x)\right|<K$ is bounded we have $K^{R_{n}}\left|\Lambda_{n}\right| \approx \gamma$ and hence $\left|\Lambda_{n}\right| \geq \gamma e^{-R_{n} \log K}$. So if $R_{n}<\frac{-\log \epsilon}{2 \log K}$ then $\left|\Lambda_{n}\right|>\gamma \sqrt{\epsilon}$. Since we have exponential return time tails, $m\left(x \in \Lambda_{0}: R(x)>T\right) \leq C \theta^{T}$ for some $0<\theta<1$. Hence $m_{0}\left(\cup \Lambda_{n} \subset \Lambda_{0}:\left|\Lambda_{n}\right|<\sqrt{\epsilon}\right)<\epsilon^{\tau}$ for some $\tau \approx \frac{-\log \theta}{2 \log K}$. Choose $0<\sigma<1$ so that $\epsilon^{\sigma}>\epsilon^{\tau}+\sqrt{\epsilon}$ for sufficiently small $\epsilon>0$.

Now $T^{j}\left(W_{\rho}^{u}(z)\right)$ expands uniformly for $j=1$ to $R_{i}-r$ then makes a full crossing of the base $\Lambda_{0}$. By full crossing we mean that on the quotiented tower $T^{j}\left(W_{\rho}^{u}(z)\right)=\widetilde{\Lambda_{0}}$, where $\widetilde{\Lambda_{0}}$ is $\Lambda_{0}$ quotiented along stable manifolds. By the same argument as in the case of billiards, by uniform expansion, for sufficiently small $\epsilon>0$ for each $j<R_{i}-r, l\left\{x \in W_{\rho}^{u}(z): d\left(x, T^{j} x\right)<\epsilon\right\} \leq \tilde{C} \epsilon<C \epsilon l\left(W_{\rho}^{u}(z)\right)$ where $l$ is one-dimensional Lebesgue measure. For $j=R_{i}-r, T^{j}\left(W_{\rho}^{u}(z)\right)$ has made a full crossing and this partitions $W_{\rho}^{u}(z)$ into components $C_{k}$ such that for each $C_{k}, T^{R_{i}-r} C_{k}$ crosses $\Lambda_{k}$. By bounded distortion, (except for a set of measure less than $\epsilon^{\tau} l\left(W_{\rho}^{u}(z)\right)$ corresponding to those $C_{k}$ such that $T^{R_{i}-r} C_{k}$ crosses $\Lambda_{k}$ and $\left.\left|\Lambda_{k}\right|<\sqrt{\epsilon}\right)$ each $C_{k}$ satisfies $l\left(T^{R_{i}-r} C_{k}\right)>\sqrt{\epsilon}$. If $l\left(T^{R_{i}-r} C_{k}\right)>\sqrt{\epsilon}$ then $l\left(y \in C_{k}: d\left(y, T^{R_{i}-r} y\right)<\epsilon\right) \leq \sqrt{\epsilon} l\left(C_{k}\right)$. This proves $l\left(y \in W_{\rho}^{u}(z): d\left(T^{R_{i}-r} y, y\right)<\epsilon\right) \leq \epsilon^{\sigma} l\left(W_{\rho}^{u}(z)\right)$ as $\epsilon^{\sigma}>\sqrt{\epsilon}+\epsilon^{\tau}$.

Each set $T^{R_{i}-r} C_{k}$ expands uniformly under $T^{s}$ until $s=R_{k}$, so for $s<R_{k}, l\left(y \in C_{k}\right.$ : $\left.d\left(y, T^{s+R_{i}-r} y\right)<\epsilon\right) \leq \sqrt{\epsilon} l\left(C_{k}\right)$. When $s=R_{k}, T^{s} T^{R_{i}-r} C_{k}$ has made a full crossing and $C_{k}$ is partitioned into sets $A_{j}^{k}$ such that $T^{s} T^{R_{i}-r} A_{j}^{k}=\Lambda_{j} \cap T^{s} T^{R_{i}-r} C_{k}$. By bounded distortion except for a set of $A_{j}^{k}$ 's of measure less than $\epsilon^{\tau} l\left(C_{k}\right)$, each $A_{j}^{k}$ satisfies $l\left(T^{s} T^{R_{i}-l} A_{j}^{k}\right)>\sqrt{\epsilon}$ in which case $l\left(y \in A_{j}^{k}: d\left(y, T^{s+R_{i}-r} y\right)<\epsilon\right) \leq \sqrt{\epsilon} l\left(A_{j}^{k}\right)$. Thus $l\left(y \in C_{k}: d\left(y, T^{s+R_{i}-r} y\right)<\epsilon\right) \leq \epsilon^{\sigma} l\left(C_{k}\right)$ for $0 \leq s \leq R_{k}$.

Given $t$ we induce a partition of $W_{\rho}^{u}(z)$ by writing, for each $x \in W_{\rho}^{u}(z), t=R(x)-r+R(f x)+$ $\ldots+R\left(f^{n(x)}(x)\right)+k(x)$, so that $F^{t}(x)$ has made precisely $n$ returns to the base and moved $k$ levels up the Tower. This defines a partition of $W_{\rho}^{u}(z)$ into intervals $I_{t}^{j}$ such that points in $I_{t}^{j}$ have not been separated on the Tower for $n-1$ returns to the base $(n(x)$ a random variable), then made a full crossing and moved up to level $k(x)$. By bounded distortion and the same argument as in the case of the components $C_{k}$, for each $I_{r}^{j}, l\left(x \in I_{r}^{j}: d\left(x, T^{r} x\right)<\epsilon\right) \leq \epsilon^{\sigma} l\left(I_{r}^{j}\right)$. This proves that for all $j, l\left(y \in W_{\rho}^{u}(z): d\left(y, T^{j} y\right)<\epsilon\right)<\epsilon^{\sigma} l\left(W_{\rho}^{u}(z)\right)$.

Now let $x \in M$ be a generic point so that for a (sufficiently small) local unstable manifold $W_{\eta}^{u}(x)$ we have $l\left(y \in W_{\eta}^{u}(x): d\left(y, T^{j} y\right)<\epsilon\right)<\epsilon^{\sigma} l\left(W_{\eta}^{u}(x)\right)$. We consider $W_{\eta}^{u}(x)$ as a measure space equipped with 1-dimensional conditional Lebesgue measure $m_{x}$.

Let

$$
E_{k}:=\left\{y \in W_{\eta}^{u}(x): d\left(T^{j} y, y\right) \leq \frac{3}{\sqrt{k}} \text { for some } 1 \leq j \leq(\log k)^{5}\right\}
$$

We have shown that for any $\delta>0$, for all sufficiently large $k, m_{x}\left(E_{k}\right) \leq k^{-\sigma+\delta}$. For simplicity, we will take $m_{x}\left(E_{k}\right) \leq k^{-\sigma / 2}$.

4.2.2. Control the conditional measure on an unstable leaf of the set of points whose neighborhoods have large overlaps with $E_{k}$, and extend these estimates to $\mu$. Define the Hardy-Littlewood maximal function $M_{l}$ for $\phi(y)=1_{E_{l}}(y)$ so that

$$
M_{l}(p):=\sup _{a>0} \frac{1}{2 a} \int_{I_{a}(p)} 1_{E_{l}}(y) d m_{x}(y)
$$


where $I_{a}(x)=\left\{y \in W_{\eta}^{u}(x): d(y, p) \leq a\right\}$. By the Hardy and Littlewood Theorem [27, Theorem 2.19], for any $C>0$,

$$
m_{x}\left(\left|M_{l}\right|>C\right) \leq \frac{\left\|1_{E_{l}} \rho\right\|_{1}}{C}
$$

where $\|,\|_{1}$ is the $L^{1}$ norm with respect to $m_{x}$. Let

$$
F_{k}:=\left\{z \in W_{\eta}^{u}(x): m_{x}\left(I_{k^{-\gamma / 2}}(z) \cap E_{k^{\gamma / 2}}\right) \geq\left(k^{-\gamma \beta / 2}\right) k^{\gamma / 2}\right\} .
$$

Then $F_{k} \subset\left\{M_{k^{\gamma / 2}}>k^{-\gamma \beta / 2}\right\}$ and hence

$$
m_{x}\left(F_{k}\right) \leq m_{x}\left(E_{k^{\gamma / 2}}\right) k^{\gamma \beta / 2} \leq C k^{-\gamma \sigma / 4} k^{\gamma \beta / 2} .
$$

If we take $0<\beta<\sigma / 4$ and $\gamma>8 / \sigma$ then for some $\delta>0 m_{x}\left(F_{k}\right)<k^{-1-\delta}$. This implies that

$$
\sum_{k} m_{x}\left(F_{k}\right)<\infty
$$

and hence by the Borel-Cantelli lemma for $m_{x}$ a.e. $x_{0}$ there exists $N\left(x_{0}\right)$ such that $x_{0} \notin F_{k}$ for all $k>N\left(x_{0}\right)$.

Since $x$ was arbitrary and the invariant measure is carried on unstable manifolds this implies that for $\mu$ a.e. $x$ there exists an $N\left(x_{0}\right)$ such that $m_{x_{0}}\left\{y \in W_{k^{-\gamma / 2}}^{u}\left(x_{0}\right): d\left(f^{j} y, y\right)<\right.$ $\frac{3}{\sqrt{k^{\gamma / 2}}}$ for any $\left.j=1, \ldots,\left(\log k^{\gamma / 2}\right)^{5}\right\}<\left(k^{\gamma / 2}\right)^{-1-\delta}$ for all $k \geq N\left(x_{0}\right)$. As in the case of billiards, since $\lim _{k \rightarrow \infty}\left(\frac{k+1}{k}\right)^{\gamma / 2}=1$, we obtain the same estimate for all $k$ sufficiently large, not just along the subsequence $k^{\gamma / 2}$.

If $d(y, z)<\frac{1}{\sqrt{k}}$ and $z \in W_{\eta}^{s}(y)$ then $d\left(f^{j} z, z\right)<\frac{1}{\sqrt{k}}$ implies that $d\left(f^{j} y, y\right)<\frac{3}{\sqrt{k}}$ since $d\left(f^{j} y, y\right) \leq$ $d\left(f^{j} z, z\right)+d(z, y)+d\left(f^{j} z, f^{j} y\right)$. Thus $d\left(f^{j} y, y\right)>\frac{3}{\sqrt{k}}$ for all $j=1, \ldots,(\log k)^{5}$ implies that $d\left(f^{j} z, z\right)>$ $\frac{1}{\sqrt{k}}$ for all $j=1, \ldots,(\log k)^{5}$ for all $z \in W_{\frac{1}{\sqrt{k}}}^{s}(y)$.

Since the holonomy map satisfies the quantitative estimates of Proposition 4.1 on each unstable manifold $W_{\eta}^{u}(x)$ in a neighborhood of $x_{0}$ of diameter $\frac{1}{\sqrt{k}}$ for sufficiently small $k$,

$m_{x}\left\{y \in W_{1 / \sqrt{k}}^{u}(x): d\left(f^{j} y, y\right)<\frac{1}{\sqrt{k}}\right.$ for any $\left.j=1, \ldots,(\log k)^{5}\right\}<\sqrt{k}^{-1-\delta}\left(1+\sqrt{k}^{-1 / 3}\right)<\sqrt{k}^{-1-\delta^{\prime}}$

for some $\delta^{\prime}>0$. Thus the fractional conditional measure on each unstable manifold in a $\frac{1}{\sqrt{k}}$ neighborhood of $x_{0}$ of points $y \in W_{\eta}^{u}(x)$ such that $d\left(f^{j} y, y\right)<\frac{1}{k}$ for any $j=1, \ldots,(\log k)^{5}$ is bounded by

$$
\left(\sqrt{k}^{-1-\delta}\left(1+(\sqrt{k})^{-1 / 3}\right)\right) / k^{-1 / 2}<\sqrt{k}^{-\delta^{\prime}} .
$$

Recalling that $u_{n}$ was chosen so that $n \mu\left(B_{e^{-u_{n}}}(x)\right) \rightarrow e^{-v}$, we obtain that for any $1 \leq j \leq$ $(\log n)^{5}$,

$$
\mu\left(X_{0}>u_{n}, X_{0} \circ T^{j}>u_{n}\right) \leq C n^{-1-\delta^{\prime} / 2}
$$

Hence $\left.n \sum_{j=1}^{(\log n)^{5}} \mu\left(X_{0}>u_{n}, X \circ T^{j}>u_{n}\right)\right) \rightarrow 0$.

4.2.3. Account for exceedences between $(\log n)^{5}$ and $\sqrt{n}$. The argument that exponential decay of correlations implies that $n \sum_{(\log n)^{5}}^{p} \mu\left(X_{0}>u_{n}, X_{0} \circ T^{j}>u_{n}\right) \rightarrow 0$ is the same as that for billiards. This concludes the proof of Theorem 4.2. 
4.3. Lorenz maps. In this section we consider one dimensional Lorenz maps that arise from geometric Lorenz flows. The Lorenz equations

$$
\dot{x}=10(y-x), \quad \dot{y}=28 x-y-x z, \quad \dot{z}=x y-\frac{8}{3} z,
$$

were introduced in 1963 by Lorenz [25], as a simplified nonlinear model for the weather. The mathematical study of these equations began with the geometric Lorenz flows, see [17, 33] which were shown to possess a strange attractor with sensitive dependence on initial conditions. Statistical properties of the actual Lorenz equations (7) were established by Tucker [31, 32]. He showed the existence of an attractor which supports an SRB measure.

The geometrical model can be described as follows. Let 0 be an equilibrium for a smooth (at least $\left.C^{1+\epsilon}\right)$ flow $T_{t}$ on $\mathbb{R}^{3}$. For the corresponding vector field $V: \mathbb{R}^{3} \rightarrow \mathbb{R}^{3}$ suppose that the eigenvalues of $(D V)_{0}$ are real and satisfy

$$
\lambda_{s s}<\lambda_{s}<0<\lambda_{u} \quad \text { and } \quad \lambda_{u}>\left|\lambda_{s}\right| .
$$

We choose coordinates $\left(x_{1}, x_{2}, x_{3}\right)$ so that $(D V)_{0}=\operatorname{diag}\left\{\lambda_{u}, \lambda_{s s}, \lambda_{s}\right\}$ and suppose that the flow $T_{t}$ is $C^{1+\epsilon}$-linearizable in a neighborhood of 0 . After rescaling, we may suppose that the flow is linearized in a neighborhood of the unit cube. Define the cross-sections $M=\left\{\left(x_{1}, x_{2}, 1\right):\left|x_{1}\right|,\left|x_{2}\right| \leq 1\right\}$, $M^{\prime}=\left\{\left(1, x_{2}, x_{3}\right):\left|x_{2}\right|,\left|x_{3}\right| \leq 1\right\}$. The Poincaré map $T: M \rightarrow M$ (where defined) decomposes into $T=T_{2} \circ T_{1}$ where $T_{1}: M \rightarrow M^{\prime}$ and $T_{2}: M^{\prime} \rightarrow M$. Write $T(x)=T_{h(x)}(x)$ where $h: M \rightarrow \mathbb{R}^{+}$is the first return time to $M$.

It is shown in [31] that $T: M \rightarrow M$ has a topologically transitive attractor with a singular hyperbolic structure. The map $T$ has a stable foliation [31], so that for some $\epsilon>0$ there exists a $T$-invariant $C^{1+\epsilon}$ foliation into local stable manifolds (including the singular stable manifold $\left.W^{s}(0) \equiv\left\{x_{1}=0\right\}\right)$, and a constant $\lambda_{0} \in(0,1)$ such that for all $x, y$ in the same local unstable manifold and all $n \geq 1,\left|T^{n} x-T^{n} y\right| \leq C \lambda_{0}^{n}$. Taking the quotient along local stable manifolds, a $C^{1+\epsilon}$ one-dimensional expanding map is $f: \bar{M} \rightarrow \bar{M}$ obtained with a singularity at 0 . We identify $\bar{M}$ with $X=[-1,1]$. The map $f$ is a one-dimensional uniformly expanding map with a singularity at $x=0$. The one dimensional Lorenz map $f: X \rightarrow X$ satisfies the following conditions:

(L1) There exist $C>0$ and $\lambda>1$ such that for all $x \in I$ and $n>0,\left|\left(f^{n}\right)^{\prime}(x)\right|>C \lambda^{n}$.

(L2) There exist $\beta^{\prime}, \beta \in(0,1)$ such that $f^{\prime}(x)=|x|^{\beta-1} g(x)$ where $g \in C^{\beta^{\prime}}(X), g>0$.

(L3) $f$ is locally eventually onto. i.e. for all intervals $J \subset X$, there exists $k=k(J)>0$ such that $f^{k}(J)=X$.

It is shown in [36] that $f$ has an absolutely continuous invariant measure $\mu$ whose density lies in $\mathrm{BV}$, the space of functions of bounded variation. Moreover from [10], the system $(f, X, \mu)$ can be modelled by a Young tower with exponential tails. Denoting the base of the tower by $\Lambda \subset X$, we let $\mathcal{P}_{0}=\left\{\Lambda_{l}\right\}$ denote the canonical partition of $\Lambda$ into subsets $\Lambda_{l}$ with $R \mid \Lambda_{l}=R_{l}$, and $f^{R_{l}} \Lambda_{l}=\Lambda$. We let $\mathcal{P}=\left\{\Lambda_{l, j}\right\}$ denote the partition on the tower, where $\Lambda_{l, j}$ is identified with $f^{j}\left(\Lambda_{l}\right),\left(j<R_{l}\right)$. We assume there exists $\theta<1$ such that $\mu(\{R>n\})=\mathcal{O}\left(\theta^{n}\right)$.

Theorem 4.3. If $(f, X, \mu)$ is a Lorenz map then a Type I extreme value law holds for the time series of observations $X_{j}=-\log d\left(f^{j} x, x_{0}\right)$ for $\mu$-a.e. $x_{0} \in X$.

Let

$$
\mathcal{E}_{k}(\epsilon)=\left\{x \in X: d\left(f^{k} x, x\right)<\epsilon\right\}
$$

and

$$
E_{k}=\left\{x \in X: d\left(f^{j} x, x\right)<\frac{1}{k}, \text { for some } 1 \leq j \leq(\log k)^{5}\right\} .
$$

Consider also a partition on the tower that consist of monotonicity subsets under $f^{k}$. This partition will be defined as follows. Given $\mathcal{P}=\cup_{l, j} \Lambda_{l, j}$ as before, let $\mathcal{P}_{k}=\bigvee_{i=0}^{k-1} f^{-i} \mathcal{P}$. For each $I \in \mathcal{P}_{k}$ we 
have $f^{i}(I) \subset \Lambda_{l_{i}, j_{i}}$ (for $i \leq k$ ), and in particular $f^{k}(I)=\Lambda_{l_{k}, j_{k}}$ (for some $\Lambda_{l_{k}, j_{k}}$ ). For $I \in \mathcal{P}_{k}$ we have bounded distortion of $f^{i} \mid I$ for $i \leq k$.

Proposition 4.2. There exists $0<a<1,0<\tilde{\theta}<1$ such that $\mu\left(E_{k}\right)<\tilde{\theta}^{-(\log k)^{a}}$.

We begin by estimating $\mu\left(\mathcal{E}_{k}(\epsilon)\right)$ and do this by considering the intersection $\mathcal{E}_{k}(\epsilon) \cap I$ with $I \in \mathcal{P}_{k}$. Given $\delta>0$, we will estimate $\mu\left(\mathcal{E}_{k}(\epsilon) \cap I\right)$ in two separate cases: i) when $\left|f^{k}(I)\right| \geq \delta$, and ii) when $\left|f^{k}(I)\right|<\delta$. In both cases i) and ii) we then take the sum of $\mu\left(\mathcal{E}_{k}(\epsilon) \cap I\right)$ over all such $I \in \mathcal{P}_{k}$. By a suitable choice of $\delta$ we show that (for fixed $k), \mu\left(\mathcal{E}_{k}(\epsilon)\right.$ ) decays to zero as $\epsilon \rightarrow 0$. We will need precise bounds on this rate of decay.

Big images. Suppose $I \in \mathcal{P}_{k}$ and $\left|f^{k}(I)\right| \geq \delta$ for some $\delta>0$. Consider the set $I \cap \mathcal{E}(\epsilon)$ and assume the scenario where $f^{k}(I)$ fully crosses $I$. We now estimate the proportion of $I$ that intersects $\mathcal{E}_{k}(\epsilon)$. Given this scenario, $\mathcal{E}_{k}(\epsilon) \cap I \neq \emptyset$, and so we (try to) solve for values $x^{-}, x^{+}$such that

$$
\begin{aligned}
& f^{k}\left(x^{+}\right)=x^{+}+\epsilon, \\
& f^{k}\left(x^{+}\right)=x^{+}-\epsilon .
\end{aligned}
$$

If a solution exists for both $x^{+}, x^{-}$then we obtain by the mean value theorem:

$$
\left(f^{k}\right)^{\prime}(c)\left(x^{+}-x^{-}\right)=\left(x^{+}-x^{-}\right)+2 \epsilon
$$

for some $c \in\left[x^{+}, x^{-}\right]$. Hence we obtain

$$
\left|x^{+}-x^{-}\right| \leq \frac{2 \epsilon}{\left|\left(f^{k}\right)^{\prime}(c)-1\right|}
$$

and in particular

$$
\frac{\left|f^{k}\left(I \cap \mathcal{E}_{k}(\epsilon)\right)\right|}{\left|f^{k}(I)\right|} \leq \mathcal{O}\left(\frac{\epsilon}{\delta}\right), \text { if }\left|\left(f^{k}\right)^{\prime}(c)-1\right| \geq C>0
$$

We have made various assumptions, namely that $f^{k}(I)$ fully crosses $I$ and such $x^{+}, x^{-}$above exist. If either of these conditions fail then the estimate obtained above for the ratio $\mid f^{k}\left(I \cap \mathcal{E}_{k}(\epsilon)|/| f^{k}(I) \mid\right.$ is still valid. i.e. either $\mathcal{E}_{k}(\epsilon) \cap I=\emptyset$ or $f^{k}(I)$ only partially crosses $I$ thus leading to a smaller esimate for $\mu\left(\mathcal{E}_{k}(\epsilon) \cap I\right)$. So by applying bounded distortion (twice) we obtain,

$$
\frac{|(I \cap \mathcal{E}(\epsilon))|}{|I|}, \frac{\left|\Lambda_{j} \cap f^{-j}(I \cap \mathcal{E}(\epsilon))\right|}{\left|\Lambda_{l} \cap f^{-j}(I)\right|}<C\left(\frac{\epsilon}{\delta}\right) .
$$

Following [8, Section 2], let $\mu_{0}$ be the invariant measure for the induced map $f^{R}$ then

$$
\mu_{0}\left(\Lambda_{j} \cap f^{-j}(I \cap \mathcal{E}(\epsilon))\right) \leq C\left(\frac{\epsilon}{\delta}\right) \mu_{0}\left(\Lambda_{l} \cap f^{-j}(I)\right) .
$$

Since all the intervals $I \in \mathcal{P}_{k}$ are disjoint we obtain a contribution $\mathcal{O}(\epsilon / \delta) \mu_{0}\left(\Lambda_{l}\right)$ of large intervals $I \in$ $\mathcal{P}_{k}$ that are contained in each $f^{j}\left(\Lambda_{l}\right)$. Now sum over $j \leq R_{l}$ to get a contribution $\mathcal{O}(\epsilon / \delta) R_{l} \mu_{0}\left(\Lambda_{l}\right)$, and then sum again over $R_{l}$ to get a total contribution $\mathcal{O}(\epsilon / \delta)$. This gives the estimate for $\mu\left(\mathcal{E}_{k}(\epsilon) \cap\right.$ $\left.\left\{I \in \mathcal{P}_{k}:\left|f^{k}(I)\right|>\delta\right\}\right)$.

Small images. Let $\Lambda_{l} \subset \Lambda$ be such that $\Lambda_{l} \in \mathcal{P}_{0}$. In the following we let $C>0$ and $\theta_{i}<1$ $(i=0,1, \ldots)$ denote generic constants which do not depend on $k$ (via $\mathcal{P}_{k}$ ). They might depend on the constants appearing in (L1)-(L3).

Lemma 4.1. There exist $\theta_{0}, \alpha, \delta_{0}<1$ such that for all $\delta<\delta_{0}$

$$
\mu\left\{\Lambda_{l} \in \mathcal{P}_{0}:\left|\Lambda_{l}\right|<\delta\right\} \leq \theta_{0}^{(-\log \delta)^{\alpha}} .
$$

We will consider later the corresponding estimate for elements of $\mathcal{P}$ and $\mathcal{P}_{k}$. 
Proof. This proposition can be proved as follows. We divide into two cases: 1 ) where $R_{l}>\sqrt{-\log \delta}$ and 2) where $R_{l}<\sqrt{-\log \delta}$.

For case 1) we establish by exponential decay of correlations:

$$
\begin{aligned}
\mu\left(\left\{\Lambda_{l} \subset \Lambda:\left|\Lambda_{l}\right|<\delta, R_{l}>\sqrt{-\log \delta}\right\}\right) & \leq \mu\left(\left\{\Lambda_{l} \subset \Lambda: R_{l}>\sqrt{-\log \delta}\right\}\right) \\
& \leq \theta^{(-\log \delta)^{\alpha}} .
\end{aligned}
$$

For case 2), we have by assumption $\left|\Lambda_{l}\right|<\delta$ and $R_{l}<\sqrt{-\log \delta}$. Hence by the pigeon hole principal and bounded distortion there exists $i<R_{l}$ such that $f^{\prime} \mid f^{i}\left(\Lambda_{l}\right)>\mathcal{O}\left[\left(\delta^{-1}\right)^{1 / \sqrt{-\log \delta}}\right]$. Define

$$
A:=\bigcup_{m=1}^{\sqrt{-\log \delta}} f^{-m}\left\{\left|f^{\prime}(x)\right| \geq(1 / \delta)^{1 / \sqrt{-\log \delta}}\right\} .
$$

For $\delta$ sufficiently small the set $\left\{x \in X:\left|f^{\prime}(x)\right| \geq(1 / \delta)^{1 / \sqrt{-\log \delta}}\right\}$ is a neighbouhood of $x=0$. Condition (L2), and bounded variation of the density of $\mu$ imply that its $\mu$-measure is bounded by $\left(1 / \delta_{1}\right)^{c / \sqrt{-\log \delta}}$ for some $c>0$ (where the constant $c$ depends on $\beta, \beta^{\prime}$ ). Invariance of $\mu$ implies that

$$
\mu(A) \leq \sqrt{-\log \delta}\left(1 / \delta_{1}\right)^{c / \sqrt{-\log \delta}} \leq \theta_{1}^{\sqrt{-\log \delta}}
$$

for some $\theta_{1}<1$. Hence to conclude case 2) we observe that that if $\Lambda \in \mathcal{P}_{0}$ with $\left|\Lambda_{l}\right|<\delta$ and $R_{l}<\sqrt{-\log \delta}$ then $\Lambda_{l} \subset A$ and so the result follows.

Define sets $A_{\delta}$ and $B_{\delta}$ as follows:

$$
A_{\delta}:=\left\{\Lambda_{l, j} \in \mathcal{P}:\left|\Lambda_{l, j}\right|<\delta\right\}, \quad B_{\delta}:=\left\{I \in \mathcal{P}_{k}:\left|f^{k}(I)\right|<\delta\right\} .
$$

We will show that $\mu\left(A_{\delta}\right)<\tilde{\theta}^{(-\log \delta)^{\alpha}}$ (for some $\tilde{\theta}<1$ ), and use this to estimate the measure of $B_{\delta}$. We state the following.

Lemma 4.2. There exist $\tilde{\theta}_{0}, \alpha, \delta_{0}<1$ such that for all $\delta<\delta_{0}$

$$
\mu\left(B_{\delta}\right) \leq k \tilde{\theta}_{0}^{(-\log \delta)^{\alpha}} .
$$

Proof. Take $I \subset \mathcal{P}_{k}$ so that $f^{i}(I) \subset \Lambda_{l_{i}, j_{i}}$ for some $i \leq k$. The sets $I$ result from a sub-partition of $\Lambda_{l, j}$ into disjoint sets which make a number of returns to the base $\Lambda$ up to time $k$. If $k+j<R_{l}$ then $I=\Lambda_{l, j}$, otherwise if $R_{l}<k+j$ we sub-divide $\Lambda_{l, j}$ into monotonicity sub-intervals $I \subset \Lambda_{l, j}$ such that $f^{i}(I) \subset \Lambda_{l_{i}, j_{i}}$ up to time $k$. Such intervals $I$ may make up to a maximum of $k$ returns to the base $\Lambda$ (before time $k$ ).

Suppose that $\left|\Lambda_{l, j}\right|<\delta$, then by uniform expansion estimates it follows that $\left|\Lambda_{l, 0}\right|=\left|\Lambda_{l}\right|<\delta$. Thus

$$
A_{\delta} \subset\left\{\cup_{l, j} \Lambda_{l, j}:\left|\Lambda_{l}\right|<\delta\right\} .
$$

Taking measures and then using Lemma 4.1 we have:

$$
\begin{aligned}
\mu\left(A_{\delta}\right) & \leq \mu\left\{\cup_{l, j} \Lambda_{l, j}:\left|\Lambda_{l}\right|<\delta\right\} \leq \sum_{\left|\Lambda_{l}\right|<\delta} R_{l} \mu_{0}\left(\Lambda_{l}\right) \\
& \leq \sum_{\substack{\left|\Lambda_{l}\right|<\delta \\
R_{l}<\sqrt{-\log \delta}}} R_{l} \mu_{0}\left(\Lambda_{l}\right)+\sum_{R_{l}>\sqrt{-\log \delta}} R_{l} \mu_{0}\left(\Lambda_{l}\right) \\
& \leq \tilde{\theta}_{1}^{\sqrt{-\log \delta}}
\end{aligned}
$$

for some $\tilde{\theta}_{1}<1$. This gives the required estimate for $\mu\left(A_{\delta}\right)$. Now for each $I \subset B_{\delta} \cap \Lambda_{l, j}$, we have that $f^{k}(I) \subset A_{\delta}$. Given $\Lambda_{j, l}$, either $k+j<R_{l}$ and $\Lambda_{j, l} \subset A_{\delta}$ or else $R_{l}<k+j$ and we subdivide 
$\Lambda_{l, j}$ into monotonicity subintervals $I$ that eventually enter $A_{\delta}$ for some time $m \in[1, k]$. Thus

$$
B_{\delta} \cap \Lambda_{l, j} \subset \Lambda_{l, j} \cap\left(\bigcup_{m=0}^{k} f^{-m}\left(A_{\delta}\right)\right) .
$$

We apply $f^{-j}$ to this set to pull back to $\Lambda_{l}$ and then sum over all such $\Lambda_{l}$. Taking the resulting measure of this set gives:

$$
\sum_{R_{l}=0}^{\infty} \sum_{j=0}^{R_{l}-1} \sum_{m=0}^{k} \mu_{0}\left(\Lambda_{l} \cap f^{-j-m}\left(A_{\delta}\right)\right)
$$

where $\mu_{0}$ is equivalent to Lebesgue (it is the measure on the base $\Lambda_{0}$ ). We split this sum over $R_{l}$ into two parts (I), and (II). In (I) we will take $R_{l}>\tilde{q}(\delta)$ for some constant $\tilde{q}$ (which is large when $\delta$ is small) and use $\mu_{0}\left(\Lambda_{l} \cap f^{-j-m}\left(A_{\delta}\right)\right)=\mu_{0}\left\{\Lambda_{l}\right\}$. This measure will be small by integrability of $R_{l}$ wrt $\mu$. In (II) we will use $\mu_{0}\left(\Lambda_{l} \cap f^{-j-m}\left(A_{\delta}\right)\right)=\mu_{0}\left(A_{\delta}\right)$ and show that this measure is small by exponential decay of correlations.

So taking $R_{l}>\tilde{q}(\delta)$, the sub-sum (I) can be estimated as:

$$
\begin{gathered}
C_{\mu} \sum_{R_{l}=\tilde{q}}^{\infty} \sum_{j=0}^{R_{l}-1} \sum_{m=0}^{k+R_{l}} \mu\left(\Lambda_{l}\right) \leq C_{\mu} \sum_{R_{l}=\tilde{q}}^{\infty} R_{l}\left(k+R_{l}\right) \mu\left(\Lambda_{l}\right) \\
\leq C_{\mu} \sum_{R_{l}=\tilde{q}}^{\infty} R_{l}\left(k+R_{l}\right) \theta_{1}^{R_{l}} \leq C k \theta_{2}^{\tilde{q}}
\end{gathered}
$$

for $\theta_{1}, \theta_{2}<1$. Taking $R_{l}<\tilde{q}(\delta)$, the sub-sum (II) can be estimated as:

$$
\begin{gathered}
C_{\mu} \sum_{R_{l}=0}^{\tilde{q}} \sum_{j=0}^{R_{l}-1} \sum_{m=0}^{k+R_{l}} \mu\left(A_{\delta}\right) \leq C_{\mu} \sum_{R_{l}=0}^{\tilde{q}} \sum_{j=0}^{R_{l}-1} \sum_{m=0}^{k+R_{l}} \mu\left(A_{\delta}\right) \\
\leq C \tilde{q}^{2}(k+\tilde{q}) \mu\left(A_{\delta}\right) \leq C k \tilde{q}^{3} \theta_{3}^{(-\log \delta)^{\alpha}} .
\end{gathered}
$$

The proof is completed by choosing $\tilde{q}=\sqrt{-\log \delta}$.

We now complete the proof of Proposition 4.2, collecting together the estimates for the corresponding cases of either having big images or small images we obtain (for some $\theta_{4}, \alpha<1$ and generic $C>0)$ :

$$
\mu\left(\mathcal{E}_{k}(\epsilon)\right) \leq C k \theta_{4}^{(-\log \delta)^{\alpha}}+C \frac{\epsilon}{\delta} .
$$

Putting $\delta=\sqrt{\epsilon}$ gives $\mu\left(\mathcal{E}_{k}(\epsilon)\right) \leq C k \theta_{5}^{(-\log \epsilon)^{\alpha}}$. Hence

$$
\mu\left(E_{k}\right) \leq \sum_{j=1}^{(\log k)^{5}} \mu\left(\mathcal{E}_{j}\left(\frac{1}{k}\right)\right) \leq \sum_{j=1}^{(\log k)^{5}} C j \theta_{5}^{(\log k)^{\alpha}} \leq C \tilde{\theta}^{(\log k)^{a}}
$$

for some $a>0, \tilde{\theta}<1$ and for some generic constant $C>0$. This completes the proof of Proposition 4.2 .

Remark 4.3.1. In Proposition 4.2 it can be further shown that $a<1 / 2$ will be sufficient. We take $a=\frac{1}{3}$.

We use the Maximal function technique of Collet [8] to carry the pointwise result over to neighborhoods. This is equivalent to the methods adopted for the Lozi map, but due to the non-standard (sub-polynomial) asypmotitics of $\mu\left(E_{n}\right)$, we give the details. In the following $B_{r}(x)$ denotes an interval of radius $r$ centered at $x$. 
Let $0<\beta \leq \frac{1}{2}$ and let $0<\rho<1$ such that $\rho \beta<\beta / 3$. Define the set

$$
F_{k}:=\left\{\mu\left(B_{\exp \left(-k^{\beta}\right)}(x) \cap E_{\exp \left(k^{\beta}\right)}\right) \geq \mu\left(B_{\exp \left(-k^{\beta}\right)}(x)\right) \exp \left(-k^{\beta \rho}\right)\right\} .
$$

If $x \in F_{k}$ then

$$
\frac{\mu\left(B_{\exp \left(-k^{\beta}\right)} \cap E_{\exp \left(k^{\beta}\right)}\right)}{\mu\left(B_{\exp \left(-k^{\beta}\right)}\right)} \geq \exp \left(-k^{\beta \rho}\right)
$$

if we define

$$
M_{l}(x):=\sup _{r>0} \frac{1}{m\left(B_{r}(x)\right)} \int_{B_{r}(x)} \chi_{E_{l}}(y) d \mu(y)
$$

we see immediately from the definition of $M_{l}(x)$ and (11) that for every $x \in F_{k}, M_{e^{k}}(x) \geq e^{-k^{\beta \rho}}$. Hence

$$
F_{k} \subset\left\{M_{e^{k^{\beta}}} \geq e^{-k^{\beta \rho}}\right\} .
$$

A theorem of Hardy and Littlewood [27, Theorem 2.19] implies that

$$
m\left(\left|M_{l}\right|>c\right) \leq \frac{\left\|\chi_{E_{l}}\right\|_{1}}{c}
$$

Since $\frac{d \mu}{d m}$ is bounded we obtain $\mu\left(\left|M_{l}\right|>c\right) \leq C \frac{\mu\left(E_{l}\right)}{c}$. As $\mu\left(E_{l}\right) \leq \mathcal{O}(1) \tilde{\theta}^{(\log l)^{1 / 3}}$ (recall we set $a=1 / 3)$,

$$
\mu\left(F_{k}\right) \leq \mathcal{O}(1) \mu\left(E_{e^{\beta}}\right) e^{k^{\beta \rho}} \leq \mathcal{O}(1)\left(e^{\alpha k^{\beta / 3}+k^{\beta \rho}}\right)
$$

where $\alpha:=-\log \theta$ and $k$ is large enough. Since $\beta / 3>\beta \rho, \sum_{k>0} \mu\left(F_{k}\right)<\infty$. By the Borel Cantelli lemma, $\mu\left(\limsup F_{k}\right)=0$, and hence for $\mu$ almost every $x$ there exists an $N_{x}$ such that for all $k \geq N_{x}, x \notin F_{k}$.

Let $x_{0}$ be such a generic point, and let $N_{x_{0}}$ be the corresponding index beyond which $x_{0}$ does not belong to any $F_{k}$. Since $\lim _{k \rightarrow \infty} e^{(k+1)^{\beta}} e^{-k^{\beta}}=1$ the fact that we restricted to a subsequence is of no consequence and we obtain the following estimate for all $n$ sufficiently large. If $1 \leq j \leq(\log n)^{5}$, then

$$
\mu\left(\left\{X \geq u_{n}\right\} \cap\left\{X \geq u_{n}\right\} \circ T^{j}\right) \leq \mu\left(B_{e^{-u_{n}}}\right) e^{-u_{n}^{\rho}}
$$

Recalling that $u_{n}$ was chosen so that $n \mu\left(B_{e^{-u_{n}}}(x)\right) \rightarrow e^{-v}$, we get, for any $1 \leq j \leq(\log n)^{5}$,

$$
n \mu\left(\left\{X \geq u_{n}\right\} \cap\left\{X \geq u_{n}\right\} \circ T^{j}\right) \leq n \mu\left(B_{e^{-u_{n}}}\right) e^{-u_{n}^{\rho}} \leq \mathcal{O}(1) e^{-u_{n}^{\rho}}
$$

On summing over $1 \leq j \leq(\log n)^{5}$ and taking limits as $n \rightarrow \infty$ we obtain the desired result.

Finally, as in the case of Lozi maps we use exponential decay of correlations to show

$$
\lim _{n \rightarrow \infty} n \sum_{(\log n)^{5}}^{p=\sqrt{n}} \mu\left(X_{0}>u_{n}, X_{0} \circ T^{j}>u_{n}\right)=0 .
$$

From [36], this estimate is in fact simpler to check by knowledge of decay of correlations in BV. We can avoid the step of approximating charactersitic functions by Lipschitz functions.

\section{REFERENCES}

[1] V. Baladi. Positive Transfer Operators and Decay of Correlations, Advanced Series in Nonlinear Dynamics, Vol 16, World Scientific, Singapore (2000)

[2] M. Benedicks and L. S. Young. Markov extensions and decay of correlations for certain Hénon maps. Asterisque No. 261 (2000), xi, 13-56.

[3] L. Barriera, Y. Pesin and J. Schmeling, Dimension and product structure of hyperbolic measures, Ann. of Math. (2), 149 (1999), 755-783

[4] L. A. Bunimovich, Ya. G. Sinai and N. . I. Chernov, Russian Math. Surveys, 45:3, 1990, 105-152.

[5] L. A. Bunimovich, Ya. G. Sinai and N. . I. Chernov, Statistical properties of two-dimensional hyperbolic billiards, Russian Math. Surveys, 46, 1991, 47-106. 
[6] N. Chernov. Decay of correlations in dispersing billiards. Journal of Statistical Physics, 94 (1999), 513-556.

[7] N. Chernov and R. Markarian. Chaotic Billiards, Mathematical surveys and monographs, Vol 127, (2006), American Mathematical Society.

[8] P. Collet. Statistics of closest return for some non-uniformly hyperbolic systems. Erg. Th. Dyn. Syst. 21 (2001), 401-420.

[9] Collet, P. and Levy, Y.; Ergodic properties of the Lozi Mappings, Commum. Math. Phys. 93, 461-481 (1984)

[10] K. Díaz-Ordaz. Decay of correlations for non-Holder observables for expanding Lorenz-like one-dimensional maps. Discrete Contin. Dyn. Syst. 15, (2006), 159-176.

[11] D. Dolgopyat. 'Limit theorems for partially hyperbolic systems' Trans AMS 356 (2004) 1637-1689.

[12] A. C. M. Freitas and J. M. Freitas. On the link between dependence and independence in extreme value theory for dynamical systems, Stat. Probab. Lett., 78, (2008), 1088-1093.

[13] J. Freitas and A. Freitas. 'Extreme values for Benedicks Carleson quadratic maps'. Ergodic Theory Dynam. Systems, 28, (2008), no. 4, 1117-1133.

[14] J. Freitas, A. Freitas and M. Todd. Hitting Times and Extreme Values, Probab. Theory Related Fields, 147, no. $3,(2010), 675-710$.

[15] J. Freitas, A. Freitas and M. Todd. Extreme value laws in dynamical systems for non-smooth observations. Preprint, 2010.

[16] J. Galambos. The Asymptotic Theory of Extreme Order Statistics, John Wiley and Sons, 1978.

[17] J. Guckenheimer and R. F. Williams. Structural stability of Lorenz attractors. Inst. Hautes Études Sci. Publ. Math. 50 (1979) 59-72.

[18] N. Haydn, Y. Lacroix and S. Vaienti. Hitting and return time statistics in ergodic dynamical systems. Ann. Probab., 33, (2205), 2043-2050.

[19] M. Hirata. Poisson Limit Law for Axiom-A diffeomorphisms Erg. Thy. Dyn. Sys., 13 , 533-556,(1993)

[20] M. P. Holland, and M. Nicol and A. Török. Extreme value distributions for non-uniformly hyperbolic dynamical systems. To appear Transactions AMS.

[21] A. Katok and J. M. Streleyn. Invariant manifolds, entropy and billiards; smooth maps with singularities, Springer Lecture Notes in Math. 1222, (1986)

[22] M. R. Leadbetter, G. Lindgren and H. Rootzén. Extremes and Related Properties of Random Sequences and Processes, Springer-Verlag, 1980.

[23] F. Ledrappier, Dimension of invariant measures, Proceedings of the conference on ergodic theory and related topics, II (Georgenthal, 1986), Teubner-Texte, Math. 94 (1987), 116-124

[24] C. Liverani and M. Wojtkowski. Dynamics Reported. 4 (1995) 130-202.

[25] E. D. Lorenz. Deterministic nonperiodic flow. J. Atmosph. Sci. 20 (1963) 130-141.

[26] S. Luzzatto, I. Melbourne and F. Paccaut. The Lorenz attractor is mixing. Commun. Math. Phys. 260 (2005) 393-401.

[27] P. Mattila. Geometry of sets and measures in Euclidean spaces: fractals and rectifiability. Cambridge Studies in Advanced Mathematics, 44, 1995.

[28] I. Melbourne and M. Nicol, 'Large deviations for nonuniformly hyperbolic systems', Transactions of the AMS, 360, (2008) 6661-6676.

[29] Misiurewisz, M: Strange attractors for the Lozi Mappings, Non Linear Dynamics, R.G. Helleman (ed), New York, The New York Academy of Sciences 1980

[30] S. I. Resnick. Extreme values, regular variation, and point processes, Applied Probability Trust, 4, SpringerVerlag, 1987.

[31] W. Tucker. The Lorenz attractor exists. C. R. Acad. Sci. Paris 328 (1999) 1197-1202.

[32] W. Tucker. A rigorous ODE solver and Smale's 14th problem. Found. Comput. Math. 2 (2002) 53-117.

[33] R. F. Williams. The structure of Lorenz attractors. Inst. Hautes Études Sci. Publ. Math. 50 (1979) 73-99.

[34] L. S. Young. Bowen-Ruelle measures for certain piecewise hyperbolic maps, Trans. Amer. Math. Soc., 287, (1985), 41-48.

[35] L.-S. Young. Statistical properties of dynamical systems with some hyperbolicity. Ann. of Math. 147 (1998) 585-650.

[36] M. Viana. Stochastic Dynamics of Deterministic systems. Braz. Math. Colloq. 21, IMPA (1997).

URL, C. Gupta: http://math.uh.edu/ ${ }^{\sim}$ ccgupta

E-mail address, C. Gupta: ccgupta@math.uh.edu

(C. Gupta) University of Houston, Department of Mathematics, Houston, TX, 77204

URL, M. Holland: http://secamlocal.ex.ac.uk/people/staff/mph204

E-mail address, M. Holland: m.p.holland@exeter.ac.uk 
(M. Holland) University of EXETER

URL, M. Nicol: http://math.uh.edu/〜nicol

E-mail address, M. Nicol: nicol@math.uh.edu

(M. Nicol) University of Houston, Department of Mathematics, Houston, TX, 77204 